grabungsbefunden ermittelt zu haben. ${ }^{1}$ ) Die räumliche Ausdehnung dieser Kultur scheint nicht sehr gross gewesen zu sein. In der Nordschweiz beginnend, ziehen sich ihre Spuren gegen die obere Donau hin und folgen dann dem Rheintal abwärts, wo sie auf beiden Ufern bis zum Neuwieder Becken in reichlichen Resten im Elsass, Württemberg, Baden, der Pfalz, Rheinhessen, Nassau und der Rheinprovinz bis Urmitz nachgewiesen $\cdot$ ist. $^{2}$ ) Von da zieht sie sich, wie Mayen beweist, in die Eifel hinauf, ist aber z. B. in den Seitentälern der Nahe und Mosel bzw. den sie begleitenden Höhen sonst noch nicht nachgewiesen. Dagegen ist sie offenbar den Rhein abwärts gezogen, denn neuerdings hat Rademacher unzweifelhafte Pfahlbaugefässreste gegenüber Cöln auf dem rechten Rheinufer bei Wahn gefunden, welche sich im Cölner prälistorischen Museum befinden. ${ }^{3}$ ) Das ist bisher ihr nördlichster Punkt, nach Osten und Westen scheint sie sich nicht weit vom Rheintal entfernt zu haben, sondern stellt sich bei uns als eine speziell rheinische, frühneolithische Kultur dar, die dann von den höher entwickelten, weit ausgedehnten Kulturen der Bandund der Schnurkeramik verdrängt worden ist. So kennt man zwar ihre Verbreitung in der Schweiz und in Deutschland wohl schon ziemlich genau, aber ihre Herkunft ist dunkel, die Kluft zwischen ihren imposanten Festungsbauten am Rhein und deren Verwandten im ägäischen Kulturkreis lässt sich vorderhand noch nicht überbrücken.

\title{
Die Hauptergebnisse der prähistorischen Ausgrabung in Vinča im Jahre 1908
}

\author{
Von M. M. Vassits, Belgrad
}

Hierzu Tafel 7-16

\section{Beschreibung des Fundortes und seiner Kulturschicht}

Im Jahre 1908 leiteten wir die Ausgrabung am prähistorischen Fundorte Vi n č a (etwa $15 \mathrm{~km}$ von Belgrad entfernt) an der Lokalität B e lo B r do (Abb. 1).

Die Kulturschicht ist abgelagert auf dem an dieser Stelle 12 in hohen Lössufer der Donau und bildet hier eine 6-8 $\mathrm{m}$ hohe kegelförmige Erhöhung (Tell?) von ungefähr $35000 \mathrm{qm}$ erhaltener Oberfläche (Abb. 2); von dem gewachsenen Lössboden wird sie getrennt durch die einstige Humusschicht ron ungefähr $0,50 \mathrm{~m}$ Dicke.

Das Ufer wird an dieser Stelle beim Hochwasserstande abgespült, wobei

1) B. J. 110 , S. 111.

2) Vgl. ausser Reinecke a. a. 0 . auch Schumacher, Altertümer unserer h. Vorzeit. V. S. $97 \mathrm{ff}$.

3) Vgl. Mannus I, Heft 1/2 1909, \$. $83 \mathrm{f}$. 
Kulturüberreste zurückbleiben und von den dortigen Bewohnern gesammelt werden. 1) Auf diese Weise erwarb das Nationalmuseum zu Belgrad durch Kauf einc schöne, nach Tausenden zählende Sammlung, die uns zu einer systematischen Grabung an demselben Fundorte veranlasst hat.

Dic Aufgabo unserer Ausgrabung in Vinica bestand weniger darin, das nouo Material zu sammeln, als vielmehr die stratigraphischen Beobachtungen einzelner Erscheinungen systematisch durchzuführen. Dadurch ebenso wie durch die zur Verfügung gestellte Bodenfläche war die Ausgrabungsmethode in voraus bestimmt. Diese Bodenfläche betrug etwa $40 \times 5 \mathrm{~m}$ und wurde daher in kleinere Teile von $2 \times 5 m$ oder $1 \times 5 m$ eingeteilt, welche dann nacheinander bis zum gewachsenen Boden abgegraben und durchgesucht wurden. Dabei ging man schichtenweise vor in Abständen von $10 \mathrm{zu} 10 \mathrm{~cm}$, so dass auch

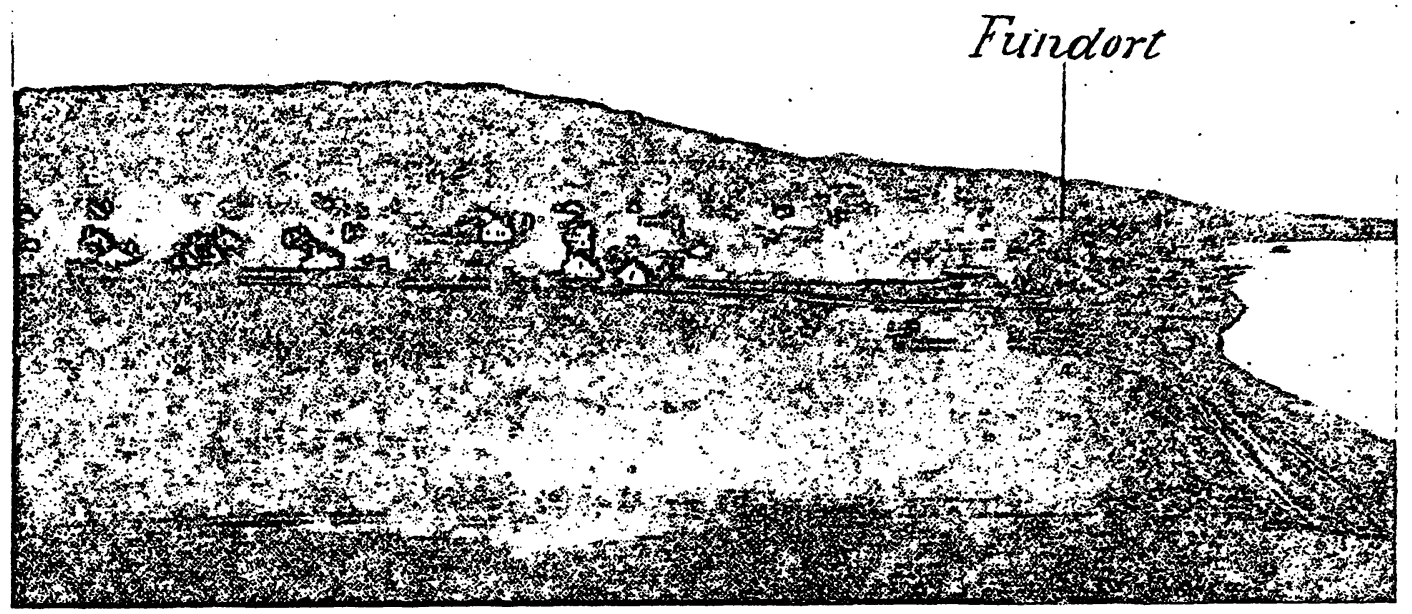

Abb. 1. Gesamtansicht von Vinča, von Süden gesehen.

das gesammelte Material nach diesem Vorgange gesondert wurde. Obzwar diese Methode in mancher Hinsicht, wie z. B. in bezug auf die Grundrissform und -Grösse der Wohnhütten mangelhaft ist, erwies sie sich dennoch als die einzig richtige, die sich unter den gegebenen Umständen anwenden liess. Denn während der Grabung stellte es sich heraus, dass in der ganzen Kulturschicht fast gar keine sterile Stelle vorhanden war. Selbst die manchmal ziemlich dicken, gestampften Fussböden enthielten Fragmente von verschiedenen Objekten und namentlich von Gefässen (Tafel 7,a).

In der Kulturschicht wurden in verschiedenen Niveaus Fussböden oder die Strata des gebrannten Lehms angetroffen. Dagegen konnte man trotz sorgfältigster Beobachtung in der Kulturschicht nicht die geringsten Spuren eines sterilen Stratums feststellen, das uns die Unterbrechung des mensch-

1) Vgl. St a ri n a r, Organ der serbischen archäol. Gesellschaft, N. S. Bd. I (1906), S. 89 bis 127; II e m n o $\mathrm{n}$, Bd. I (1907), S. 177-187. 
lichen Lebens auf diesem Wohnplatze bezeugen würde. Aber auch die Beobachtung der. Stratigraphie der einzelnen Kulturerscheinungen spricht für ununterbrochene Besiedlung des Platzes.

Von grosser Bedeutung für die Datierung dieses Fundortes ist das Vorhandensein eines oben $15 \mathrm{~m}$ breiten und nach unten sich verengenden Grabens, dessen Profil sich ganz deutlich von den horizontalen Ablagerungen der Kulturschicht unterscheiden liess (vgl. Tafel 7, a und c). Der obere Rand dieses Grabens reichte bis 0,80 $m$ bzw. 1,20 $m$ unter der jetzigen Erdoberfläche; sein unterer Teil endete an der Oberfläche des Lössbodens. Die Bedeutung dieses Grabens erklärten uns zwei kleinere ähnliche Gräben, die sich beiderseits dieser kegelförmigen Erhöhung in dem vorhandenen Profil befinden,

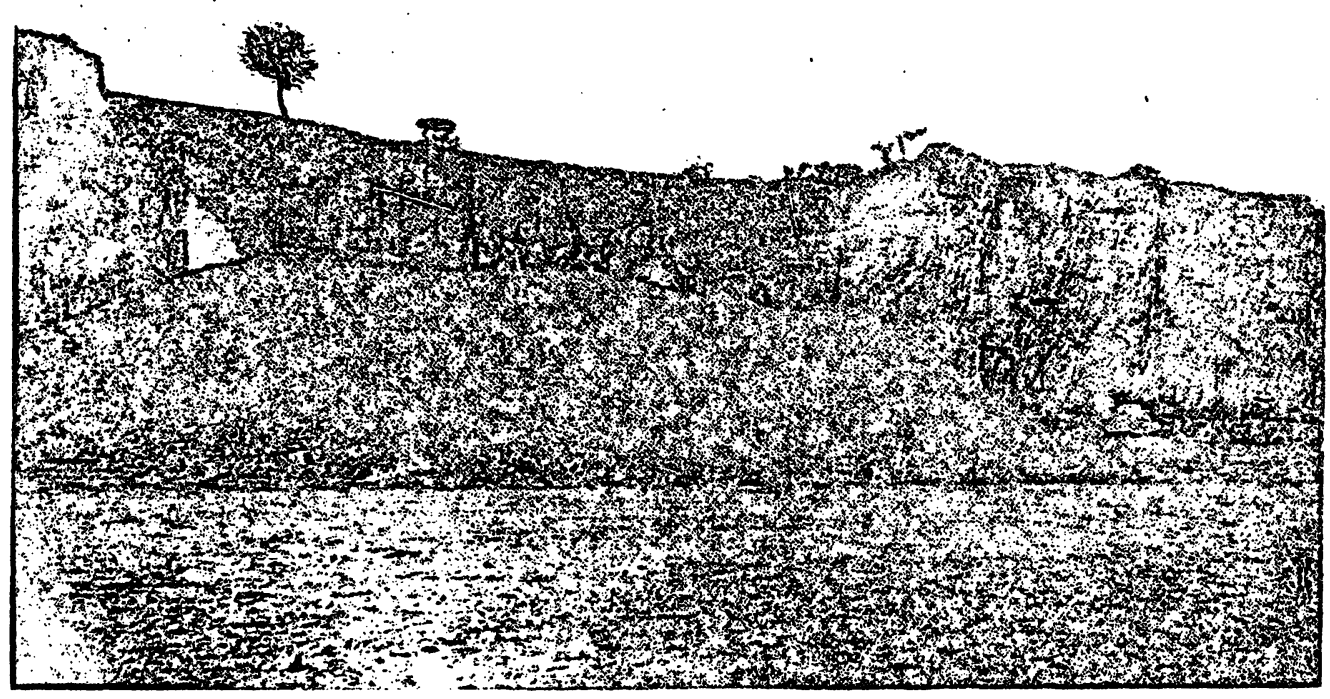

Abb. 2. Der Fundort während der Arbeit. Wand mit dem Profil der Kulturschicht.

(Vgl. Tafel 7, a.)

so dass man alle die Gräben als zur Befestigung dienend betrachten muss, ohne jetzt genauer bestimmen zu können, wie diese Befestigungsart ausgesehen haben mag. Der grosse, mittlere Graben war verschüttet und enthielt nur zerstreute Fragmente; selbst auf seinem Boden fand man Gefässscherben ron den Gattungen, die man in dem ersten Meter unter der Erdoberfläche zu finden pflegte.

Diesen Befestigungsgräben entsprechen auch die Funde aus der obersten Schicht, bis jetzt leider nur zufällige, die aber nach ihrer Art und dem Aussehen der La-Tène-Periode zuzuschreiben sind. Ähnliche Funde wie diese vom Belo Brdo wurden auch an anderen Lokalitäten in Vinča, aber auch in dem benachbarten römischen $\mathrm{T} r \mathrm{i}$ c or $\mathrm{n}$ i u m angetroffen. Die oberste Schicht auf dem Belo Brdo in Vinća muss jedenfalls stärkeren Umänderungen ausgesetzt gewesen sein, wobei auch der grossc Graben angefüllt wurde. Wann dies aber geschah, können wir jetzt nicht mehr mit Sicherheit bestimmen. 
Als terminus ante quem würde man vielleicht den Anfang des 15. Jahrhunderts n. Chr. betrachten können; damals stand an dieser Stelle eine kleine Klosterkirche, um welche dann allmählich ein altserbischer Friedhof angelegt wurde. Dic altserbischen Gräber sind auch oberhalb des verschütteten Spitzgrabens in cler Tiefe von 0,6 bis $0,7 \mathrm{~m}$ gefunden worden.

Wïhrend die oberste Schicht schwerlich sich in ihrem ursprünglichen Zustande erhalten hat, hat sich dagegen die unterste Schicht sehr gut erhalten. Dic unterste Schicht charakterisiert man am besten mit der Bezeichnung
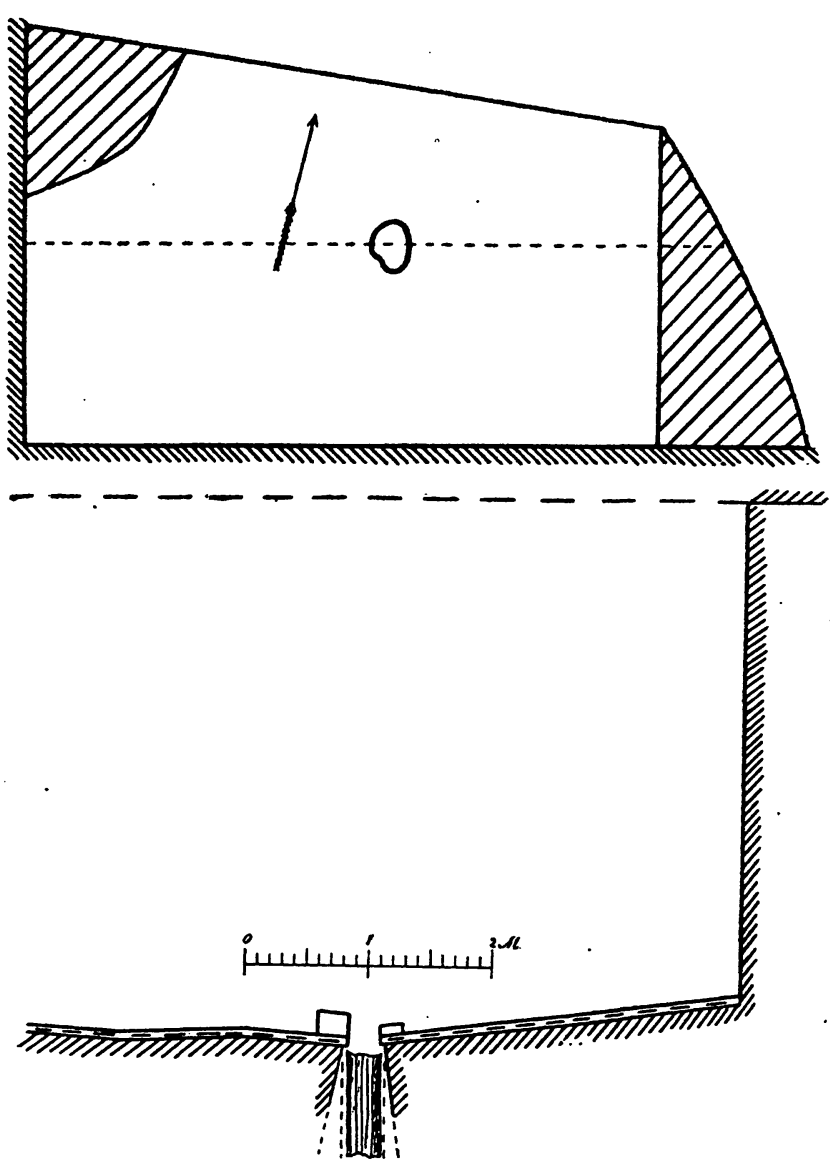

Abb. 3. Grundriss und Durchschnitt eines gestampften Lehmbodens in der Tiefe von 3,70 m mit dem Lager der Dachbalkenstütze. Wohngrubenschicht (Taf. $\bar{i}, \mathrm{~b}$ und c). Diese Gruben hatten einen mehr oder weniger kreisrunden oder auch unregelmässigen Grundriss und sind alle in den gewachsenen Lössboden gegraben, unterhalb der ursprünglichen Humusschicht, die damals die Erdoberfläch€ darstellte. Eine von diesen Gruben erreichte die Tiefe von 9,5 $m$ unter der jetzigen Erdoberfläche und scheint ziemlich grosse Dimensionen gehabt zu haben.

Von den Wohnhütten der mittleren Strata kann man vorläufig nur soviel behaupten, dass sie grössere horizontale Ausdehnung und einen mehr oder weniger viereckigen Grundriss wohl gehabt haben müssen. Nur in zwei bis drei Fällen waren auch in dieser mittleren Schichtentiefe grössere Gruben beobachtet, ohne ihre Verwendung genauer bestimmen zu können. Dagegen

wurde einmal in der Tiefe von $3,70 \mathrm{~m}$ ein weiterer gestampfter Fussboden angetroffen, in welchem ein vertikales Lager eines verfallenen Holzbalkens vorhanden war (Abb. 3). Diesen Holzbalken wird man kaum anders als als einstige Dachstütze erklären dürfen. Ausgedehntere gestampfte Fussböden traf man auch unmittelbar oberhalb der ursprünglichen Humusschicht, die fast in derselben Horizontale mit den Grubenrändern lagen.

Aus diesen kurzen Andeutungen ist leicht zu ersehen, dass die durchgeführte Grabung allen Kulturerscheinungen in Vinca nicht gerecht werden konnte. Neue Aufgaben und neue Ziele bleiben der künftigen Forschung vorbehalten. Trotzdem aber sind einige Resultate erreicht worden, die unsere volle Beachtung verdienen. 

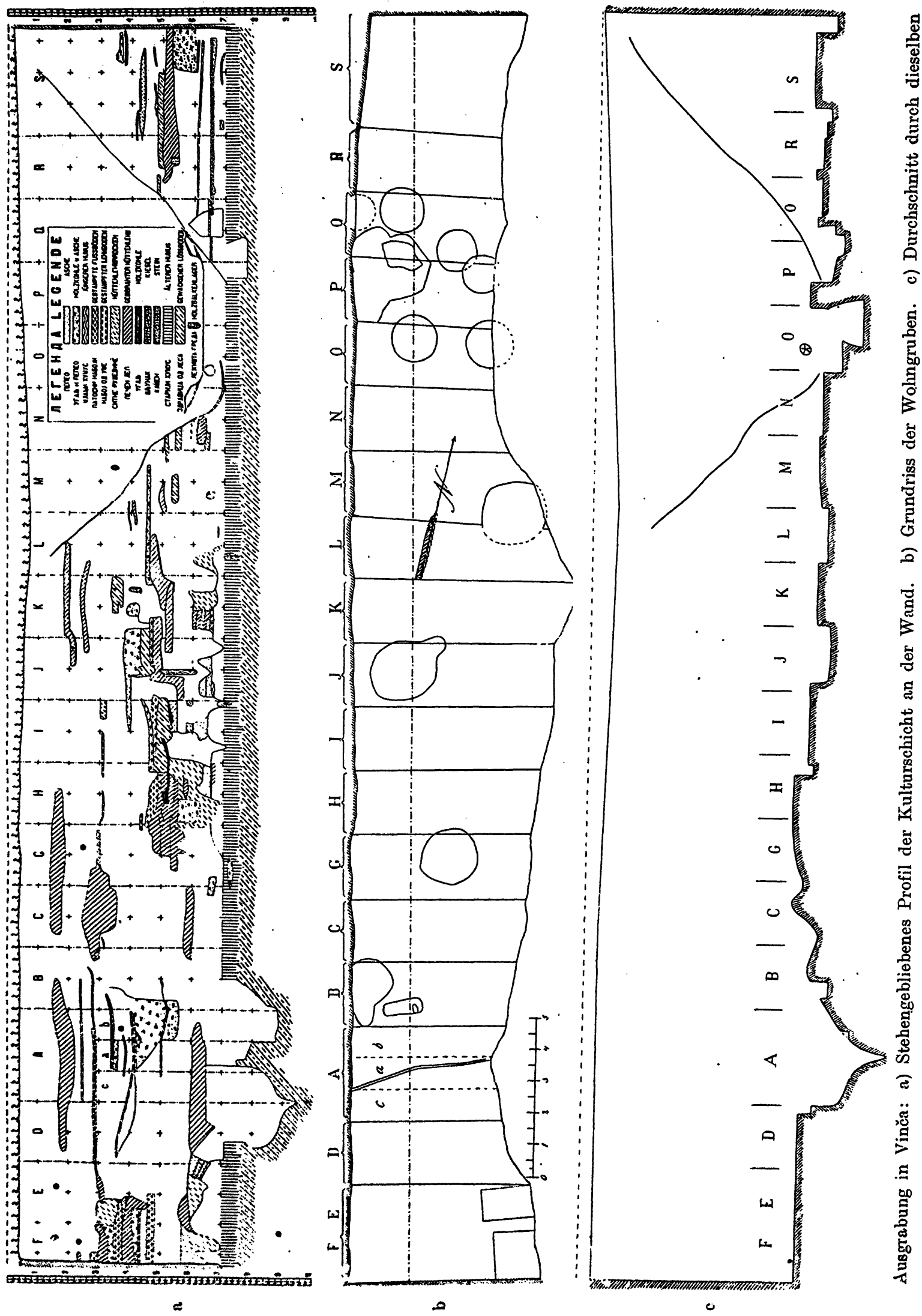

3. M. Vassits, Ausgrabung in Vinça 

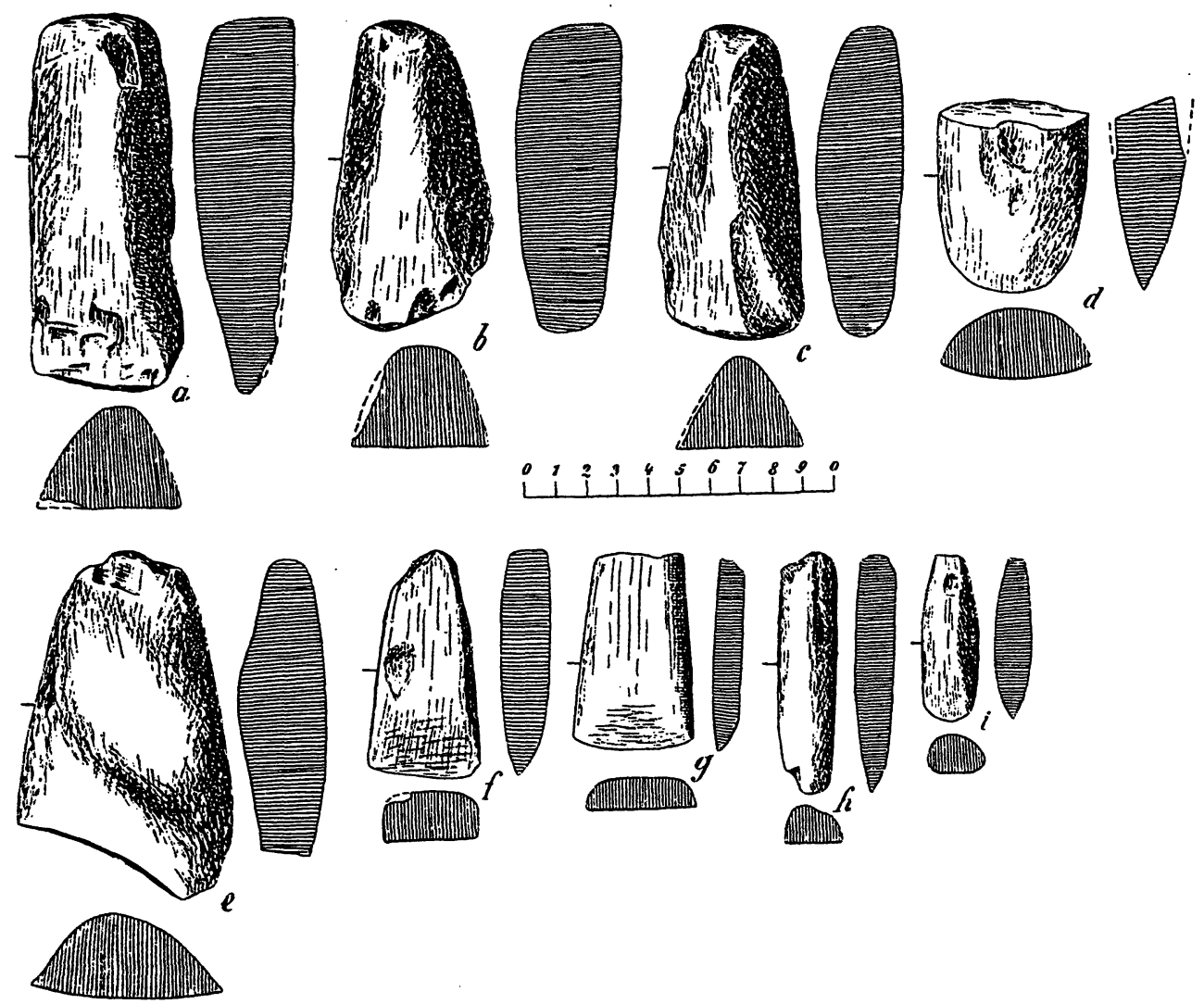

a) Steingeräte von Vinča, etrra $1 / 3$ nat. Gr. - National-NIuseum in Belgrad
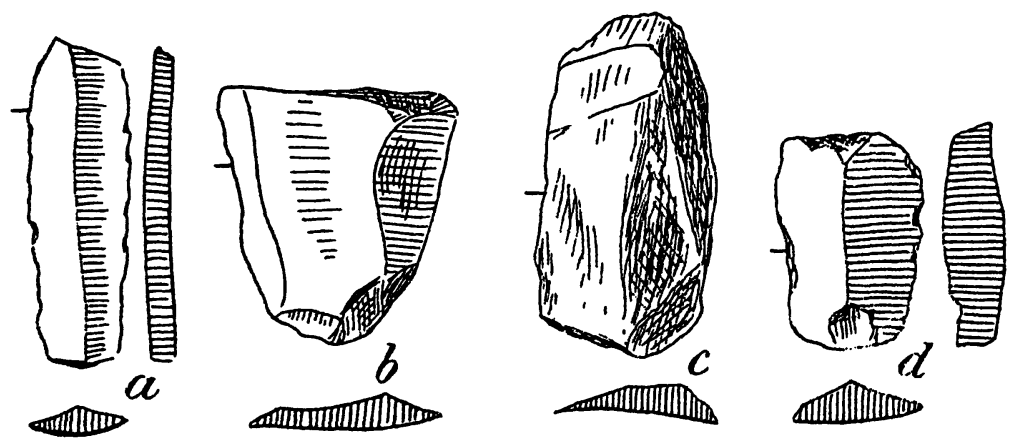

b) Geräte aus Feuerstein von Vinča, 1/1 nat. Gr. - National-Museum in Belgrad 


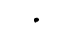




\section{Hauptfunde und einige Kulturerscheinungen in Vinča.}

Wir führen hier nur diejenigen Funde an, die einerseits für den Fundort selbst und andererseits für die gesamte prähistorische Forschung in unteren Donauländern Bedeutung haben können.

$\mathrm{Ste}$ in - und Flin twerkze u g e üblicher Form sind in allen Tiefen der Kulturschicht ron $0-9,5 m$ gefunden worden (Tafel S). O bsidianmesser und - Split ter (Abb.4) traf man in der Tiefe ron 2,6 bis $6,5 \mathrm{~m}$. Die $\mathrm{Hirsch}$ ornhar punen (Abb. 5) und ihre Fragmente erschienen in der Tiefe von 2-5,8 m. $\mathrm{Zu}$ gleicher Zeit wurden auch die $\mathrm{Hirsch}$ -

hornh a cken (Abb. 6) mit dem schrägen Schaftloche gefunden.

Die T onstatu e t t e $n$ sind in zahlreichen und verschiedenen Bildungen in allen Schichtentiefen gefunden worden (Taf. 9). Sie lassen sich, wie wir sehen werden, in mehrere Typen einteilen, sei es nach der Körperhaltung der Figur, sei es nach der Art der
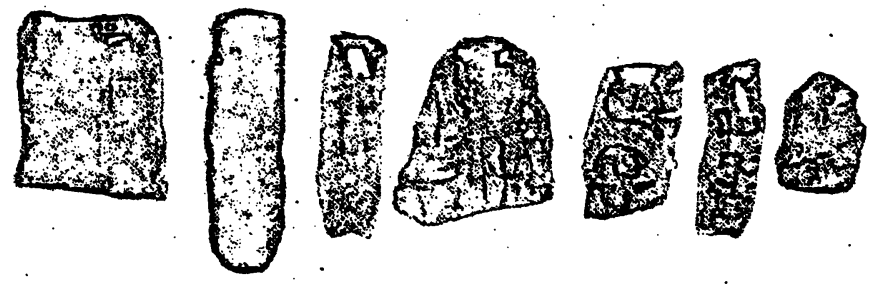

Abb. 4. Obsidiangeräte ron Vinča, $4 / 5$ nat. Grösse. - NationalMruseum in Belgrad.

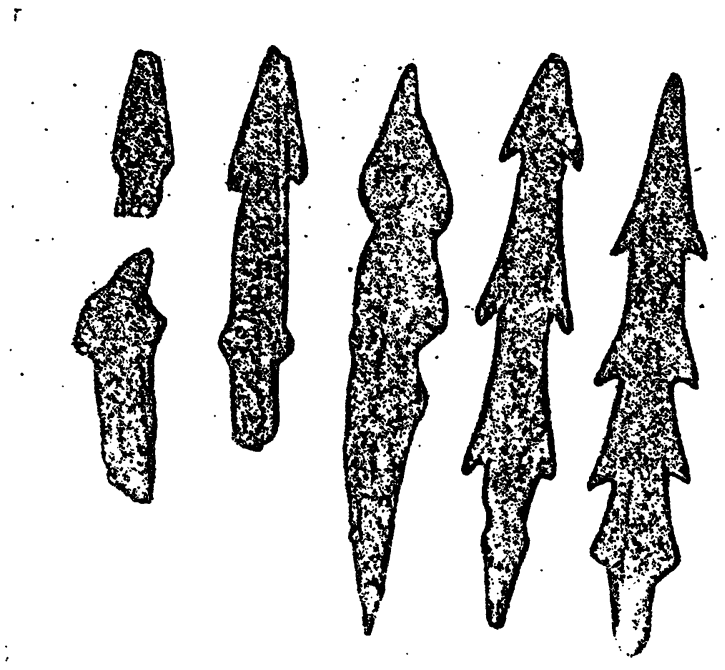

Abb. 5. Hirschhornharpunen von Vinča, 1/2 nat. Grösse. National-XIuseum in Belgrad.

Körperteile. Die steatopygischen Figuren kommen in den tiefsten Schichten vor (einmal in der Tiefe von $9,5 \mathrm{~m}$ ), wo auch eine eigentümliche Art der Gesichtsbildung erscheint. Die Gesichtsfläche ist nämlich etwa dreieckig, und in der Witte dieser Fläche sitzt eine plastische, warzenförmige Nase, an deren Seiten manchmal eingeritzte gerade Winkel - Augen - sich befinden. Derartige Gesichtsbildung ist in Serbien bis jetzt nur in den tieferen Schichten von Vincia angetroffen worden (Taf. 9, a). Jie mittleren Strata enthalten Tonstatuetten mit mehr Detail in der Körperbildung ebenso wie in der Tracht- und Schmuck- 
darstellung (Taf. 9, b-f). Rohere und degenerierte Darstellungen erscheinen schon in den mittleren, aber hauptsächlich in den oberen Schichten (Taf. 9, g). Zum ersten Male in Serbien ist bei dieser Grabung eine $\mathrm{m}$ ä $\mathrm{n}$ li c h e T o $\mathrm{n}$ statuet te ('Taf. 9, h) in der Tiefe von $6 \mathrm{~m}$ gefunden worden.

Der bulgarischen und trojanischen Analogien wegen verdienen hervorgehoben zu werden ein Fragment der Statuette aus Knochen ('Iaf. 9, i), welches in der Tiefe von $5,3 \mathrm{~m}$ gefunden ist. Dieses Fragment rührt. von einer rohen und ganz summarischen Darstellung des menschlichen Körpers her.

Gegenstände aus weissem Kalkstein und Marmor sind in Serbien bis jetzt fast ausschliesslich in Vinča gefunden worden. Es

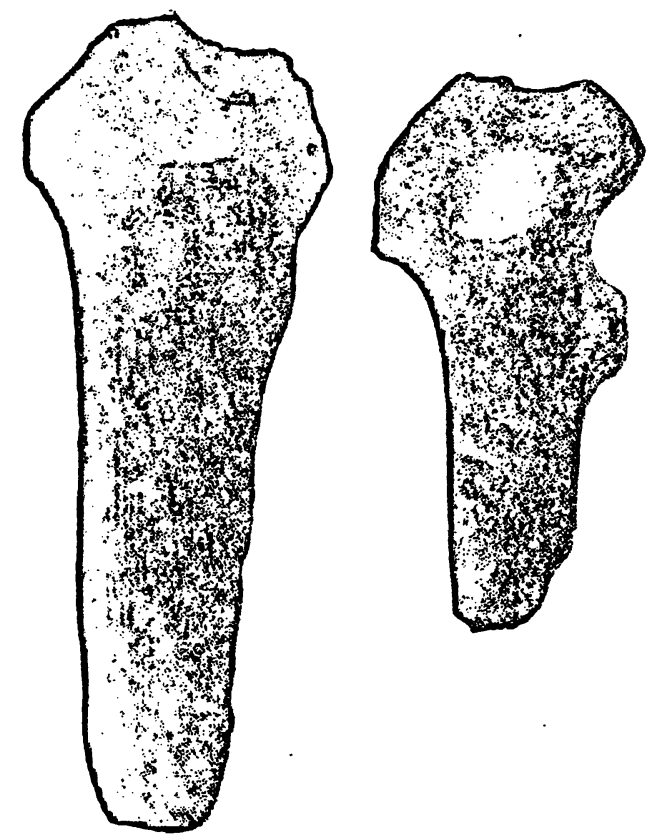

Abb. 6. Hirschhornhacken von Vinča, $1 / 3$ nat. Grösse. - National-Museum in Belgrad. kommt nur einmal (in der Tiefe von $3,10 \mathrm{~m}$ ) der kleine Rumpf eines Vierfüsslers (Ochsen?) vor; die übrigen Gegenstände sind meistens A n hängse 1, die mit einer Öse zum Durchziehen der Schnur versehen sind (Abb. 10, a). Derartige Objekte erschienen in der Tiefe von 1,8 bis $7,2 \mathrm{~m}$.

Die Muschelringe (Taf. 10, b) und Anhängsel (vgl. Taf. 10, a), oben rechts) aus $\mathrm{Muschelschale}$ sind ebenso gefunden. Solche Objekte und ihre Fragmente erschienen in der Tiefe von $1,4-7,8 \mathrm{~m}$.

Die Form und der im Voraus bestimmte Umfang des vorliegenden Berichtes verbieten uns eine ausführlichere Aufzählung der bezeichnenden keramischen Erscheinungen von Vinča. Dies bleibt der speziellen Publikation vorbehalten. Wir beschränken uns hier auf einige Bemerkungen.

Selbst die tiefsten Stellen der Wohngruben lieferten Gefässfragmente von hoher technischer Vollendung; die Glättung der Gefässoberfläche kann man mänchmal nicht anders als'vollendet bezeichnen. Es ist aber kein Fragment vorhanden, das die Kenntnis der Drehscheibe verraten würde.

Unter den Gefässformen zeichnet sich zunächst eine sehr zahlreich vertretene Fus s s h a le aus; die in der Grösse verschieden sein kann, jedoch immer einen höheren, vollen Fuss hat, an welchem sie bequem beim Tragen gefasst werden kann (Taf. 11, a). Der bezeichnendste Teil dieser Fussschale ist eben der Fuss, der sich gut erhalten konnte und der auch zahlreich gefunden wurde. Derartige Füsse kamen nur in vier Fällen in den höheren Schichten vor $(1,82,12,6$ und 2,9 $\mathrm{m})$. Sonst wurden sie regelmässig in der Tiefe von 3,9 bis $8,2 m$ gefunden.

Ebenso bezeichnend ist auch die a $\mathrm{n}$ hropomorphe $\mathrm{V}$ as e: mit 


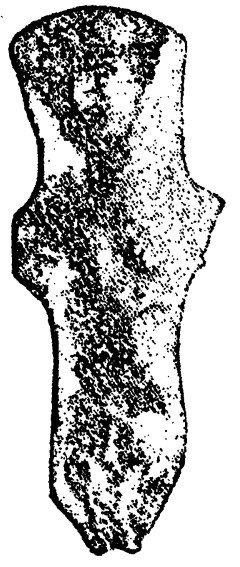

$\mathbf{a}$

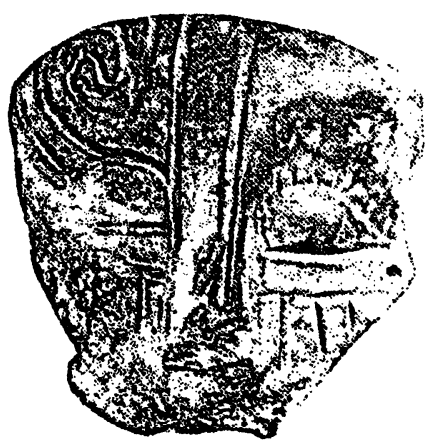

b

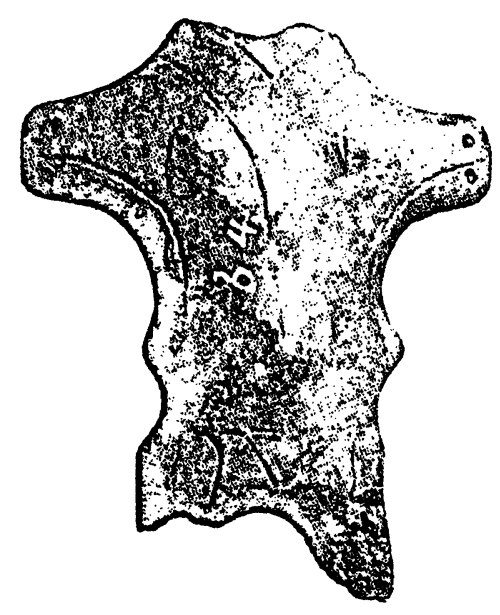

c

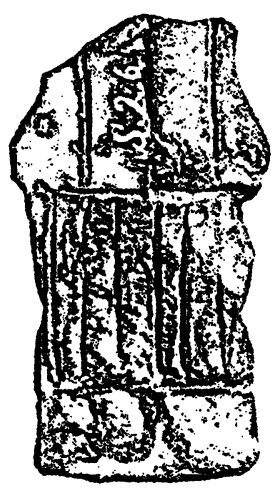

d

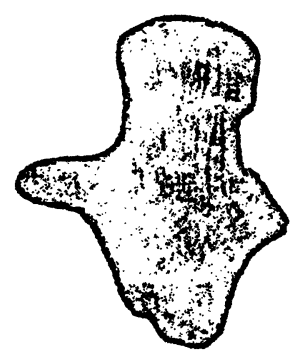

e

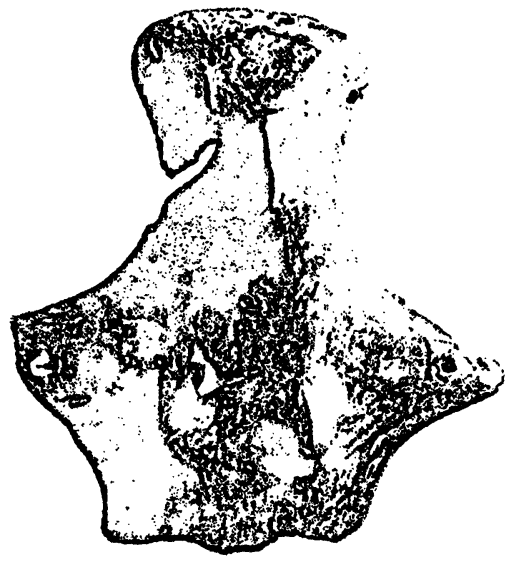

g

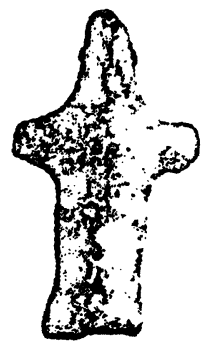

h

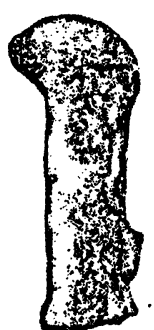

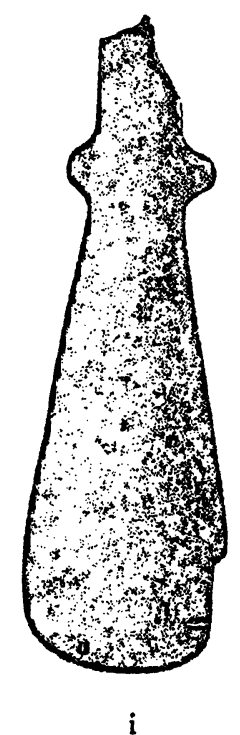

Tonstatuetten von Vinča. a) und d) 3 nat. Gr., b, (e, e-i $2 / 3$ nat. Gr. - National-Museum in Iielgrad

M. M. Vansitm. Ausgrabung in Vitüa 


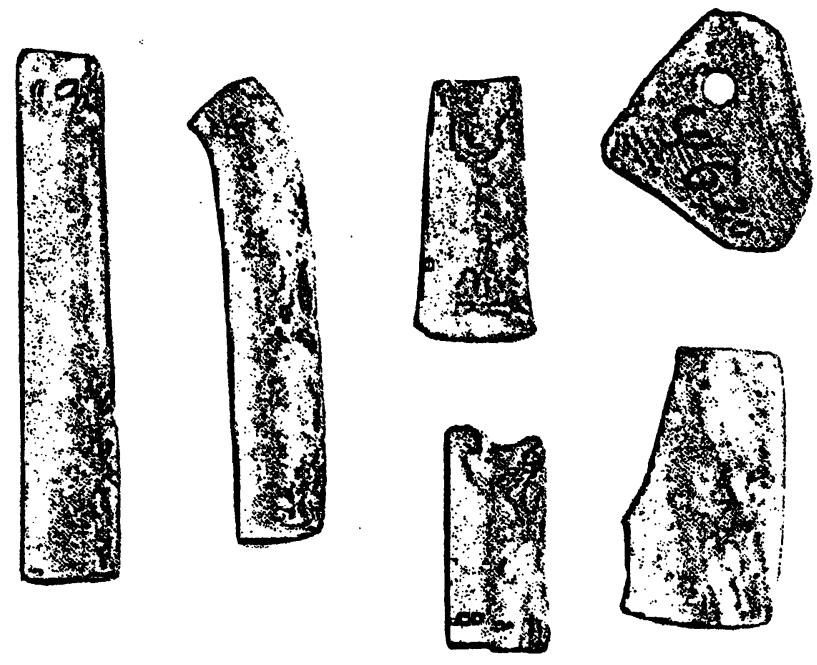

a) Anhänger aus treissem Kalkstein, Marmor und Muschelschale, 3/4 nat. Gr. National-Museum in Belgrad.

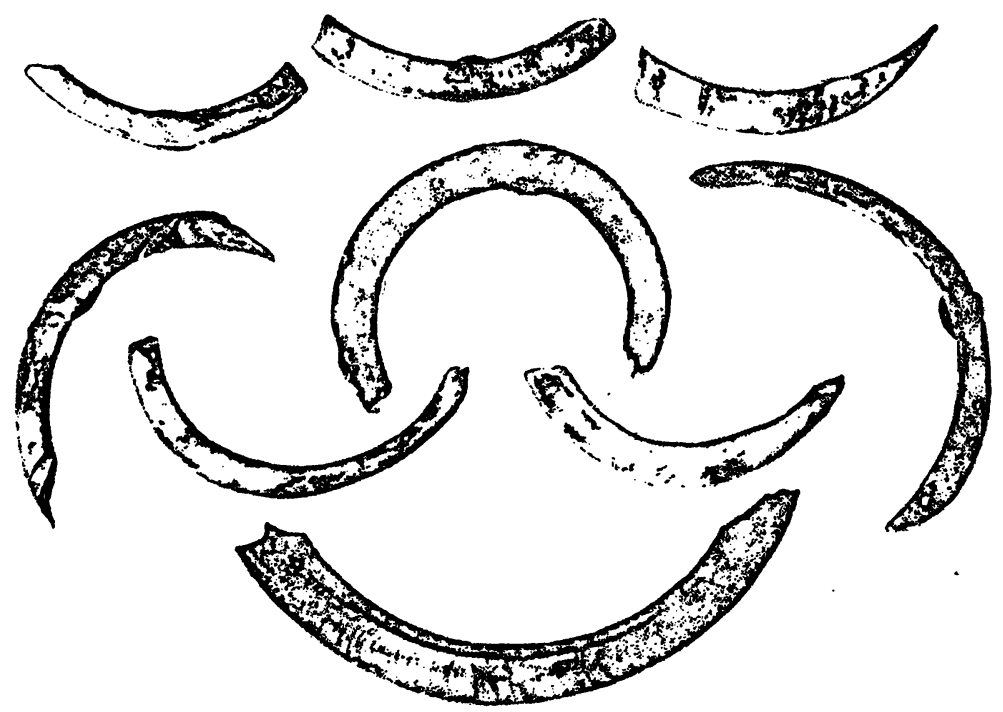

b) Muschelringe. $3 / 4$ nat. Gr. - National-Museum in Pelgrad 



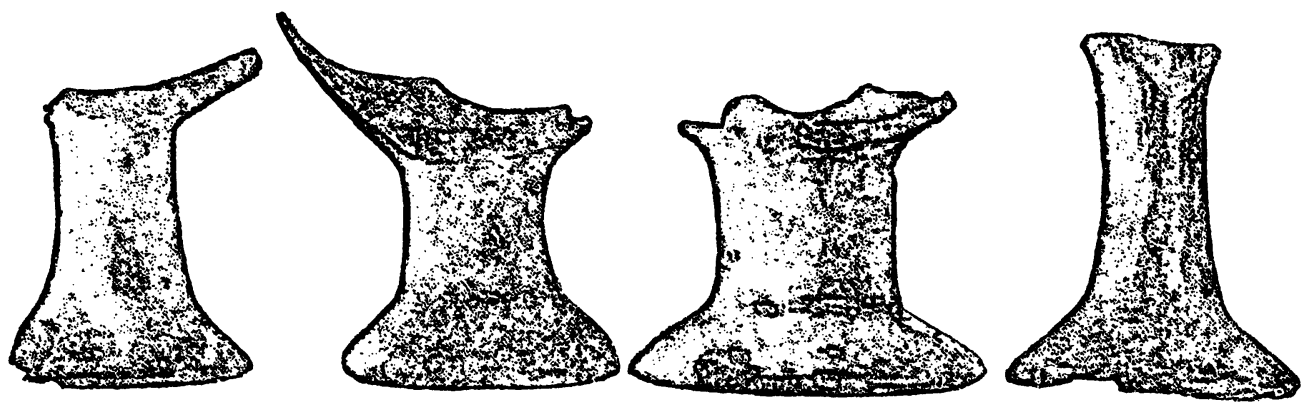

a) Untere Teile von Fussschalen, $1 / 3$ nat. Gr. - National-Mluseum in Belgrad
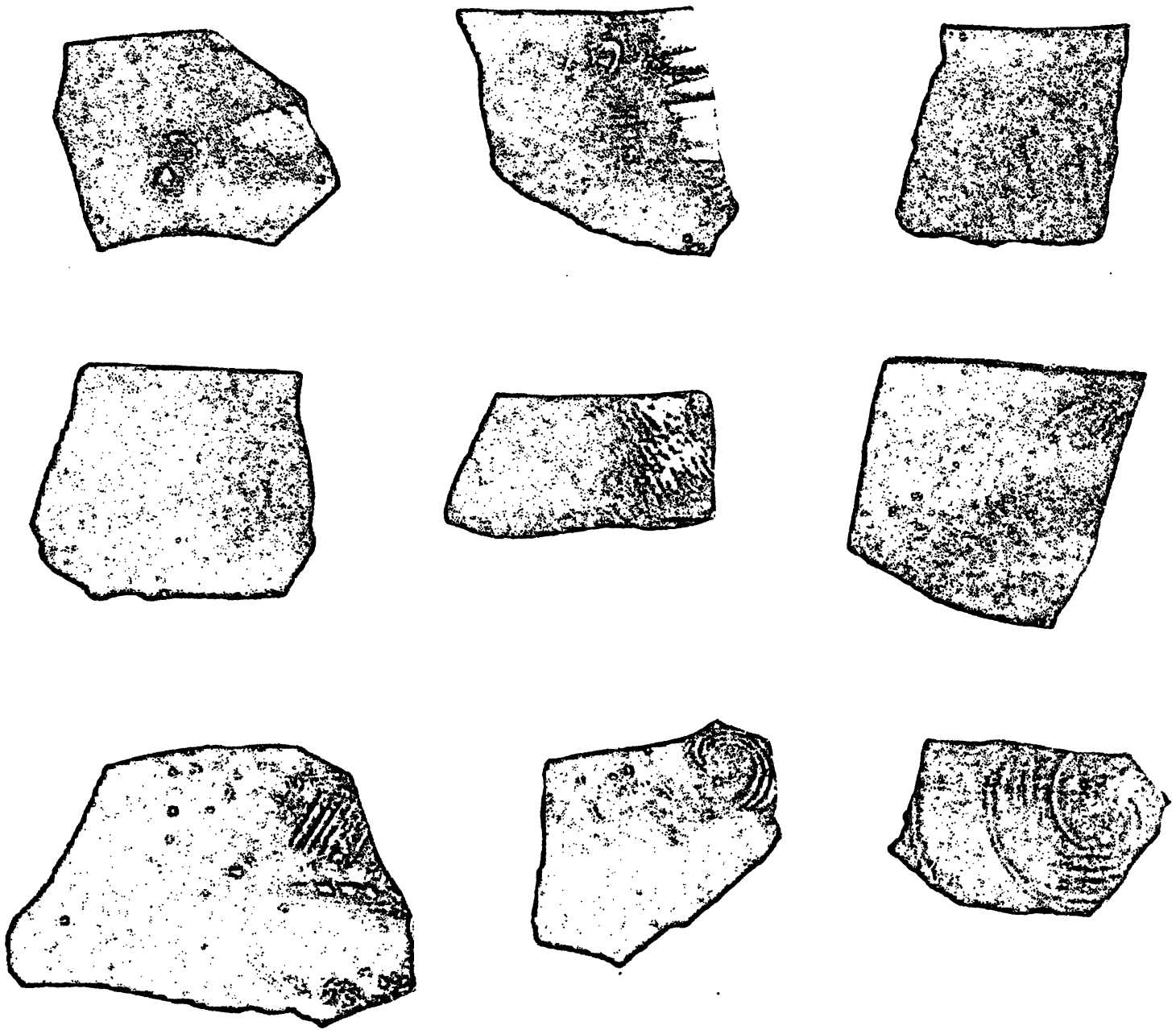

b) Scherben von Gefäissen mit cingedrückten Ornamentun, ${ }_{3}^{1}$ nat. (ir. - National-Museum in lielgrad 
der Gesichtsdarstellung auf dem Gefässhalse (Abb. 7, weniger zahlreich) und auf dem Gefässdeckel (Abb. 8). Nur zweimal (in der Tiefe von 2 und $3 \mathrm{~m}$ ) w urden die Fragmente derartiger Gefässe in den höheren Schichten gefunden. Dagegen sehr zahlreich kamen solche Fragmente in der Tiefe von 4,6 bis $8,2 m$ vor.

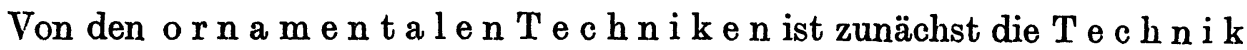
der eingedrückten Ornamente (Taf. 11, b) zu nennen, welche uns aus Serbien schon von mehreren Fundorten her bekannt ist. ${ }^{1}$ ) Bezeichnend für diese Technik ist ihr Erscheinen in den tiefsten und mittleren ebenso wie in den allerjüngsten Schichten in Vinča.

Eine zweite ornamentale Technik ist diejenige, $n$ ach welcher a uf weisslichgrau überzogener Gefässoberfläche die schwarzen, glänzenden Glättmusterdurch die Beseitigung des Überzuges bergestellt werden (Taf. 12 und 13). Auch diese Technik ist uns schon aus Serbien bekannt; ${ }^{2}$ ) in Vinča erscheint sie in der untersten Wohngrubenschicht und entwickelt sich parallel mit der Technik der eingedrückten Ornamente bis zum Schlusse der Periode der jüngsten Schicht.

Die Ornamente der üblichen Ritzte c h n ik (Taf. 14) trifft man in der Tiefe von 2,5 bis $9,5 m$. Auf den Scherben aus den tieferen Schichten sind die eingetieften Ornamente in der Regel leer (Taf. 14, a-c); die weisse Inkrustation kommt erst in den mittleren Schichten vor (Taf. 14, d).

Mit der Fussschale ist auch der rote $\ddot{U}$ b e r z ug stets verbunden (Taf. 11,a). Er ist fast regelmässig geglättet, manchmal ohne jegliche Glättungsspuren. Ein derartiger Úberzug erscheint auf keiner anderen Gefässgattung in Vinča; dass er aber absichtlich zur Gefässverzierung verwendet wurde, beweisen uns einige Fragmente, an welchen der Überzug mit dem Pinsel oder mit einem Stück Stoff aufgetragen war.

Zum ersten Male in Serbien wurde in Vinča bei dieser Ausgrabung auch die Technik der roten Mattmalerei (Taf. 15, a) festgestellt. Sie ist zunächst eine sekundäre und auch sicher jüngere Erscheinung in der Kulturschicht von Vinča. Diese Technik besteht darin, dass die Gefässe mit eingedrückten oder eingeglätteten Ornamenten nachträglich nochmals mit einzelnen Streifen, ja sogar mit ganzen Mustern in dieser Technik der roten Mattmalerei verziert werden. Die Streifen und Muster werden immer mit

1) Vgl. LXX. Glas Srpske Kraljerske Akademije Nauka, 1906, S. $211 \mathrm{ff}$.

2) Vgl. LXX. Glas Srpske Kraljevske Akademije Nauka, 1906, S. $237 \mathrm{ff}$. 
roter Farbe ausgef ührt; sie erscheinen matt und lassen sich leicht wegwischen. - Nebst den Kannen werden in dieser Technik hauptsächlich die Schalen mit dem hochaufgerichteten, manchmal besonders profilierten Rande verziert. Fragmente dieser Technik erscheinen in der Tiefe von 0,70 bis $4,6 m$. - Vergleicht man abor diese Schichtentiefen mit denjenigen der Fussschale und des mit ihnen verbundenen roten Úberzuges, so wird man diese rote Mattmalerei als j ü n g o r o Erscheinung betrachten müssen. Die Ưbergriffe dieser beiden 'Tochniken über ihre durchschnittliche Erscheinungsgrenze sind recht bezeichnend für die Kontinuität der Besiedlung, die auch durch andere Erscheinungen bezeugt wird.

Die grosse Zahl der gefundenen Fragmente mit rotem Úberzuge einerseits und derjenigen mit der roten Mattmalerei andererseits berechtigt uns, die in diesen Techniken |verzierten Gefässe als lokales einheimisches, in

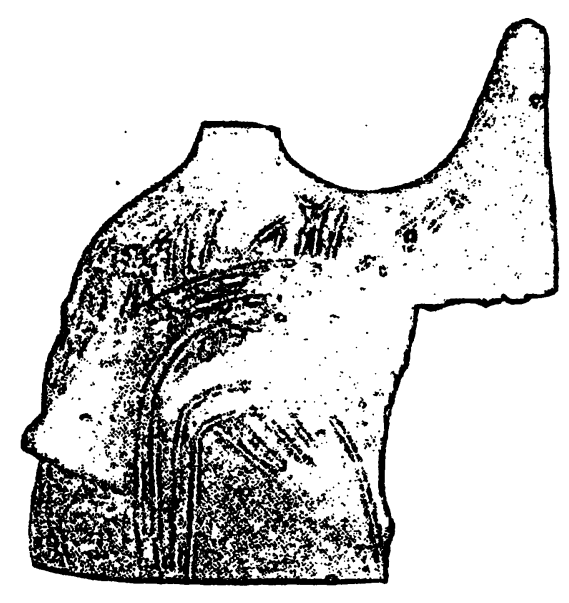

Abb:8. Deckel mit Gesichtsdarstellung, $1 / 2$ nat. Grösse. - National-Museum in Belgrad. Vinča selbst entstandenes Produkt zu betrachten. Ganz anders steht es in dieser Hinsicht mit zwei anderen Gegenständen besonderer Art, die wir als kleine drei- oder vierfüssige Altärchen bezeichnen möchten. Im ersten Falle, in der Tiefe von $4,8 \mathrm{~m}$, wurde ein solcher Gegenstand gefunden, der einen matte $n$ b läulichen Überzug hatte. Im zweiten Falle, in der Tiefe von 5,6 m, ist ein dreifüssiges Altärchen (Abb. 9) gefunden worden. Oberhalb der beiden erhaltenen Füsse befinden sich die üblichen Ansätze in Form des $\mathrm{Ochsen-}$ kopfes. Diese Ochsenköpfe, wie auch das ganze Altärchen, sind mit einem bräunlich-gelblichen matten Überzuge überzogen, a uf welchem die matten braunroten Ornamente erscheinen.

Die grosse Bedeutung des letztgenannten Stückes ist klar. Es ist zunächst eine einzig dastehende Erscheinung und daher kaum als lokales einheimisches, in vinča hergestelltes Produkt, sondern viel eher als I m p o r tst ü ck aus irgend welchem, leider nicht sicher zu bestimmenden Orte zu betrachten. Die Feststellung dieser Erscheinung ist sehr wichtig für die richtigere Beurteilung anderer in Vinča einheimisch gewordener Erscheinungen, deren Analogien, ja Vorbilder, uns in weiter entfernte Gebiete führen.

In technischer wie dekorativer Beziehung sind noch einige Besonderheiten an den Gefässen zu erwähnen, die uns das Verhältnis einer ganzen Fundortgruppe zu Vinča erklären können. - Die r o t e Inkru s t a t i o n der eingeritzten Ornamente ist nur einmal, und zwar in der Tiefe von $3,4 m$ angetroffen. - Die eingeritżten Ornamente sind in den jüngeren Schichten selten; statt ihrer kommt eine besondere Art solcher vor, 

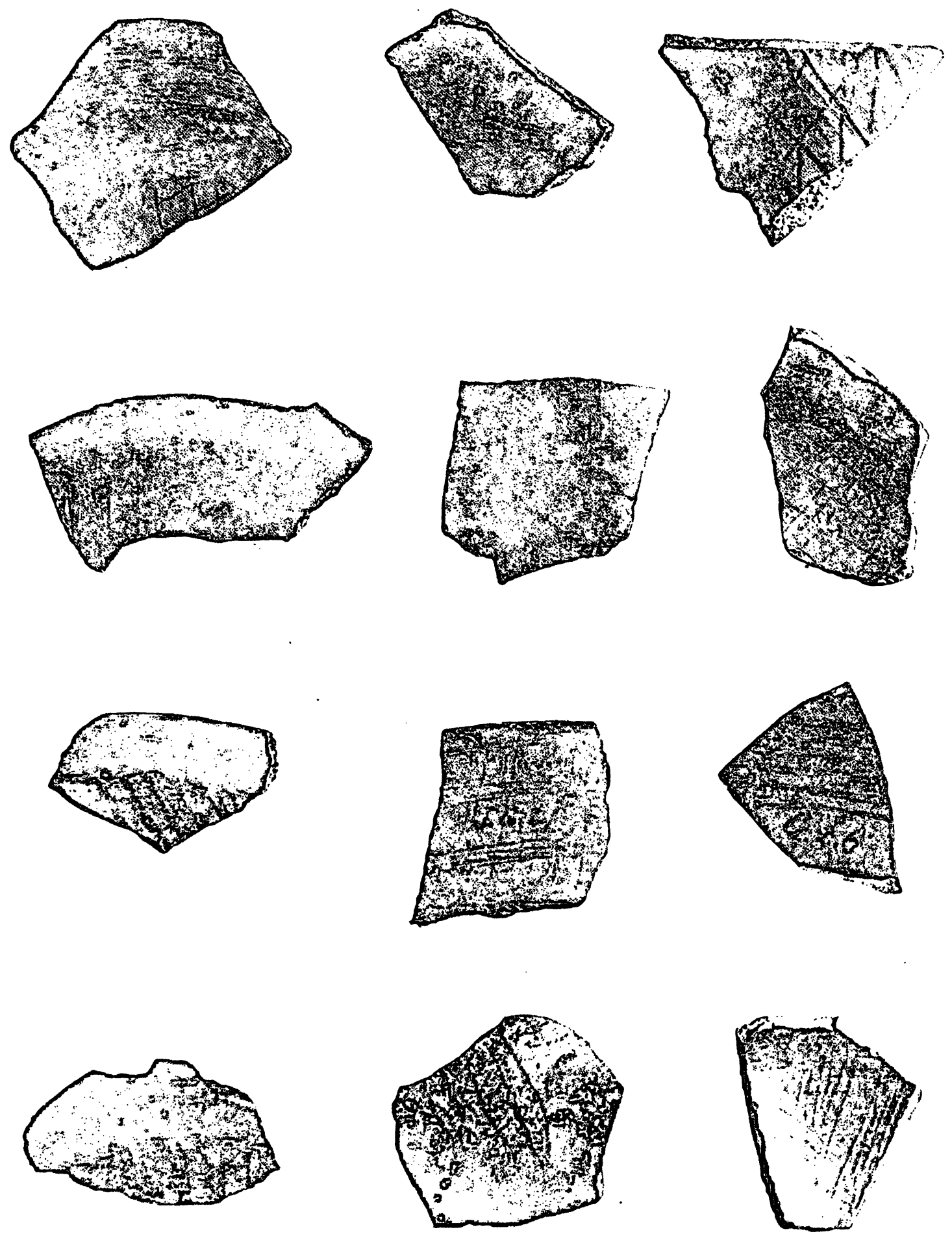

Scherlsen von Gefässen mit schwarzen, geglätheten Ornamenten auf grauweisslich ïber\%ogener Oberfläche, ca. $1 / 2$ nat. Gr. - National-.lluseum in Belgrarl 

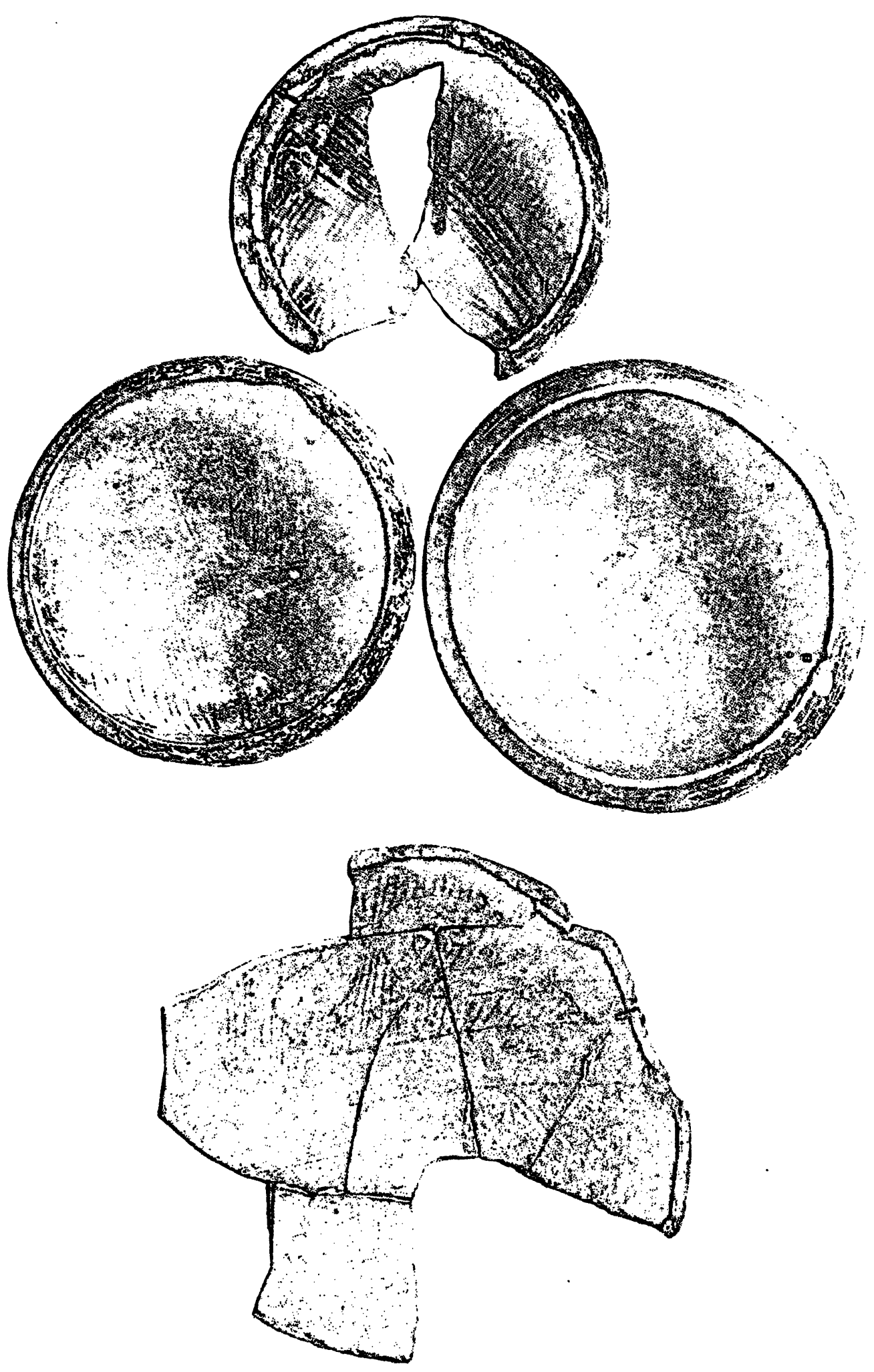

Schalen mit schwarzen geglätteten Ornamenten auf grauwcisslich ülberzogener Oberflïche, $1 / 2$ nat. Gr. - National-Mluseum in Pelgrad

M M Vassitw. Ausyrabung in Vinča 

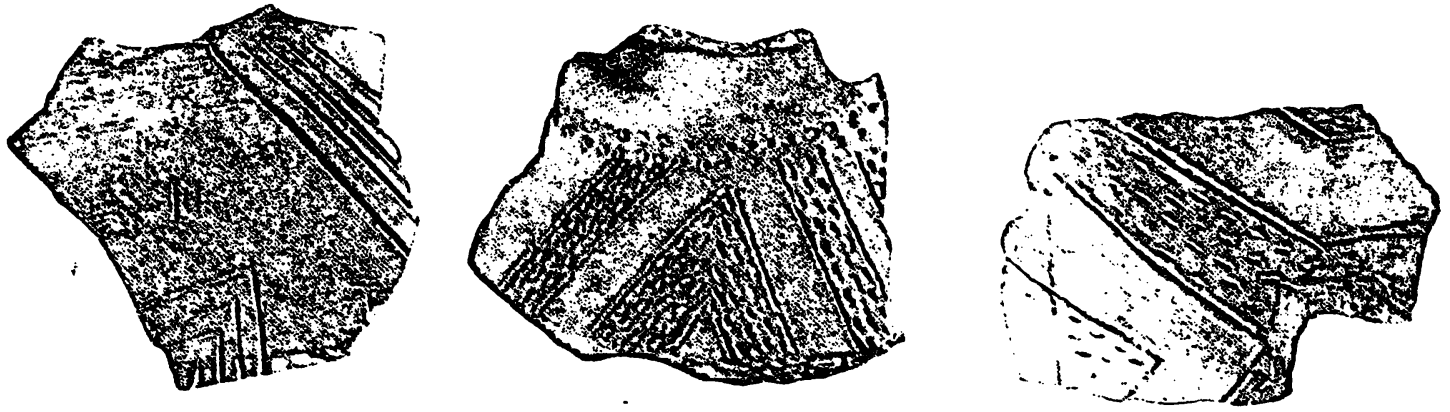

b
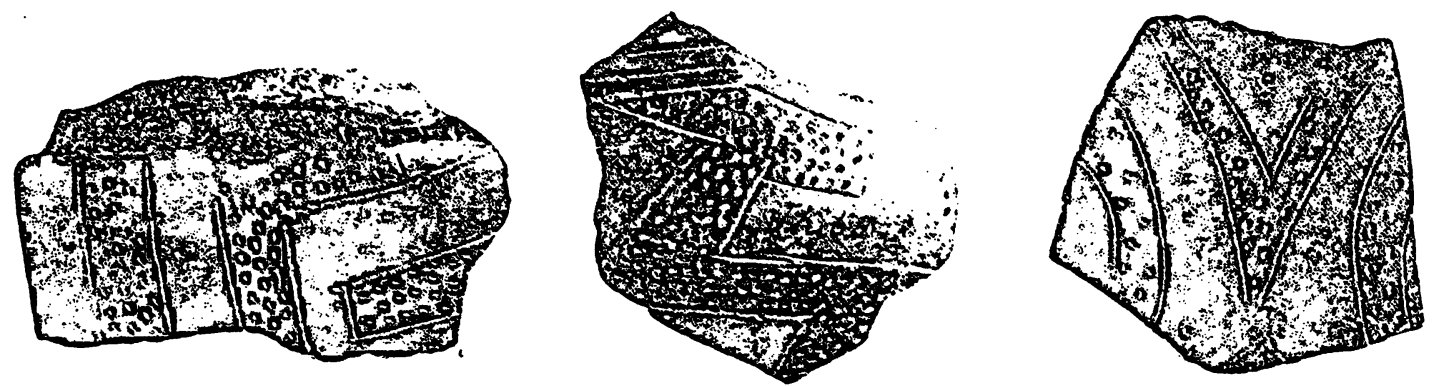

c
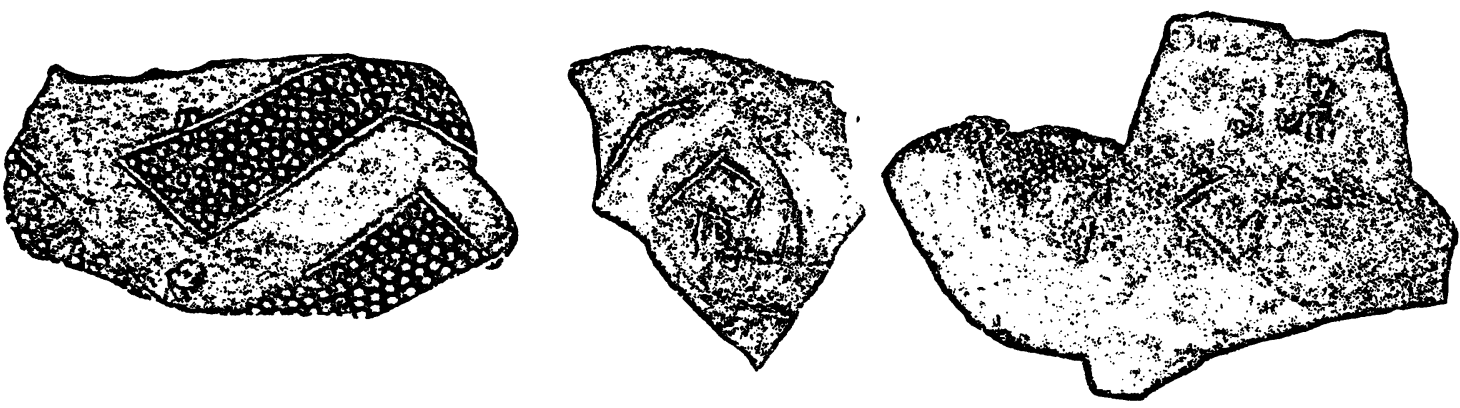

d
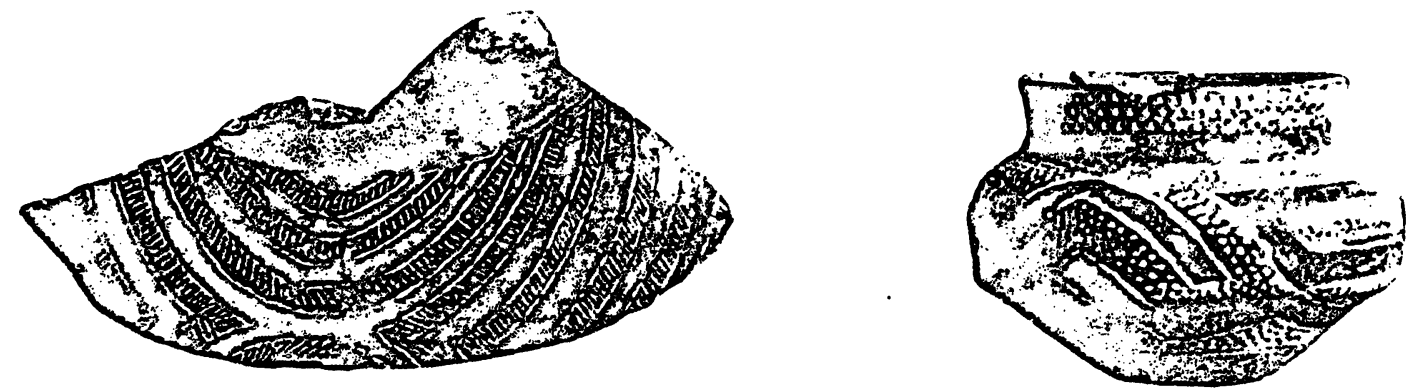

a-c) Scherben von (iefässen mit eingerit_ten Ornamenten. b) Bruchstück und restauricrtes (Gefitiss mit eingeritzten weiss inkmistierten Verzierungen, etwa : nat. Gr. - Natimal-1/useum in Belgrad 
bei welchen die $\mathrm{Z}$ eich $\mathrm{nung}$ in den weichen Ton mit einem mehr oder weniger stumpfen Instrumente eing e ti eft wird (Taf. 15, b). Solche Beispiele sind gefunden worden in der Tiefe von 0,9 bis 2,3 $\mathrm{m}$. - Die nach dem Prinzipe des sog. „R a hmenstil e s" (Taf. 15, c) verzierten Gefässe kamen vor in der Tiefe von 0,6 bis 2,2 $\mathrm{m}$. Die „Stichkanaltechnik” (Taf. 15, d) erscheint gleichfalls in den obersten Schichten von 1,5 $\mathrm{m}$ aufwärts. Klein $\theta$ W a r z e n oder B u c k el (vgl. Taf. 15, b) an den Gefässwänden erscheinen in der Tiefe von 0,5 bis $3,3 \mathrm{~m}$.

Es seien noch einige charakteristische Erscheinungen auf den Gefässen erwähnt. Die Abdrückevon Schilfgeflechten an der unteren Bodenfläche kamen vor in der Tiefe von 4,5 bis $6,2 \mathrm{~m}$. Die pi ctogrophischen Zeichen oder Marken (Taf. 16) erscheinen, in der Tiefe ron 0,6 bis $5,4 \mathrm{~m}$, aber auch in der Wohngrubenschicht.

Die ornamentalen Motive sind durchaus geometrisch. Die Spirale kam vor in der Tiefe von 2,5 bis $6,8 \mathrm{~m}$. Die „e ckig e" $\mathrm{Sp}$ ir a le wurde angetroffen in den Tiefen von 2,5 bis $5,5 \mathrm{~m}$.

Zu den Fundobjekten, die für Vinča eine besondere Bedeutung haben, rechnen wir auch die Erze. Ein Stück des Bleierzes, Galenit, ist gefunden worden in der Tiefe von $2,2 \mathrm{~m}$. Die Erzstücke, welche $\mathrm{C} i \cdot \mathrm{n} \mathrm{n}$ b a rit enthalten, sind angetroffen in der Tiefe von $3,6,4,1,4,6$, 4,9 und $5,6 \mathrm{~m}$.

Das Erscheinen des Erzes auch in den tieferliegenden Strata setzte auch das Vorhandensein der Metallobjekte auf diesem Fundorte voraus. Und in der Tat, die sorgfältige Durchsuchung der ausgegrabenen Kulturschicht bestätigte vollauf unsere Erwartungen. So ist in der Tiefe von $1 \mathrm{~m}$ ein Stückchen des spiralförmig gerollten Metalldrahtes gefunden worden. Kleine Brocken von oxydiertem Metall (Kupfer?) wurden wiederholt (nicht weniger als in zwanzig Fällen) gefunden, und zwar in der Tiefe von 1,5, 1,7, $2,2,1,2,2,2,3,2,4,2,5,2,6,2,8,2,9,3,2,5,1,5,4,6,3$ und $7,5 \mathrm{~m}$. Kleine N e t a 11 p er le n (Kupfer?), mit einem Loche zum Durchziehen der Schnur versehen, sind auch öfters gefunden worden, und zwar in der Tiefe von 2, 2,1, 2,2 (mehrere Stücke), 4,8 und 6,1 $\mathrm{m}$.

Für den Fundort Vinča ist bezeichnend, dass die Metallobjekte und -spuren fast die gleichen sind ebenso in bezug: auf die Quantität wie auch die Form (abgesehen von dem erwähnten spiralförmig gerollten Metalldraht) in den jüngeren und in den älteren Schichten. Für die Bestimmung des Alters dieses Fundortes und seiner Schichten in der Hauptsache ist dies eine wichtige Erscheinung. Ganz anders würde sich die Sache verhalten müssen, wenn die oberen, zugleich auch jüngeren Strata mehrere Netallobjekte geliefert hätten, die man in den unteren, älteren Strata nicht gefunden hätte.

Die Ausgrabung in Vinça hat uns, trotz der ungünstigen Umstände, unter welchen sie durchgeführt wurde, viele neue Erscheinungen geliefert, die bis jetzt ron keinem anderen Fundorte Serbiens bekannt waren. 


\section{Die südöstlichen Analogien zu diesen Funden und ihren Erscheinungen}

Der Vergloich der Vinćafunde mit denjenigen der südöstlich von Vinča gelegenen Fundorte hat 1. zu zeigen, wieweit in der südöstlichen Richtung dio ähnlichen oder die gleichen Erscheinungen festgestellt werden können, 2. auf Grund dieser Ähnlichkeit oder Gleichheit die Datierung der Vinčafunde nach der für die südöstlichen Fundgruppen geltenden Chronologie zu erstreben, 3. die Frage aufzuwerfen, ob die Vinča-Kultur selbständig oder von den südöstlichen Kulturkreisen abhängig ist.

Von den $\mathrm{Ste}$ i $\mathrm{n}$ wer $\mathrm{kz}$ e u g e n ist die Form des schuhleistenförmigen Steinbeiles für Vinča charakteristisch. In Bulgarien finden wir sie seltener (ein Exemplar aus der Umgebung von Varna, Kreismuseum in Varna), ebenso wie sie auch in Troja selten ist. Die übrigen Formen der Steinbeile finden ihre Analogien in Bulgarien usw.

O bs idianmesser und - S c haber sind jetzt zum ersten Male in Serbien gefunden, Analogien aus Bulgarien sind bis jetzt unbekannt; dagegen kommen sie in Troja (Katalog Nr. 8476 usw.) und Thessalien (Tsuntas, Ai

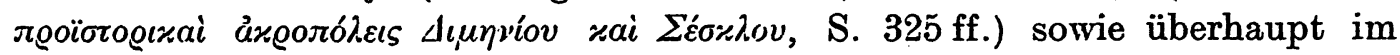
ägäischen Kreise vor.

$\mathrm{Zu}$ den $\mathrm{Hirsch}$ ornharpunen führen wir als Analogie diejenigen aus der Umgebung von Varna in Bulgarien (Kreismuseum Varna) an. Dagegen die analogen Funde aus der Schweiz, die die nächsten in nordwestlicher Richtung sind, brauchen hier gerade der grossen Entfernung wegen nicht berücksichtigt werden.

An den plastịschen Darstellungen des menschlichen Körpers aus Vinča finden wir zahlreiche und mannigfaltige Erscheinungen, die ihre nächsten Analogien .im Südosten haben.

Was das $M$ a t e r i a 1 betrifft, so ist ausschliesslich Ton ${ }^{1}$ ) zur Herstellung von Statuetten verwendet. Dem Geschlechte nach sind alle weiblich; nur einmal ist ein Mann dargestellt (Taf. 9, h). Dieser hat seine Analogien in Figuren aus Petsofa ${ }^{2}$ ) und aus Thessalien, ${ }^{3}$ ) obgleich auch in diesen Gebieten männliche Statuetten in der älteren Zeit zu den Seltenheiten gehören.

Die w e i b li c hen T ons t a t u e t t e $n$ lassen sich von verschiedenen Gesichtspunkten aus in Gruppen und Typen einteilen. Nach der Technik zerfallen sie in z.w e i G r u p p e n: A) voll und B) hohl gearbeitete Statuetten. Die Gruppe A ist charakteristisch für die älteren Strata in Vinča, ebenso wie für die übrigen serbischen Fundorte der älteren Zeit, dagegen die Gruppe B für die jüngeren Strata in Vinca und für eine ganze Anzahl Fundorte am Donauufer (vgl. das bekanntè Kličevacer Idol). Die beiden Statuettengruppen (A und B) haben ihre Analogien im Südosten, welche, was noch charakteristischer ist, dort in derselben Zeitfolge wie hier erscheinen.

1) Mit Ausnahme eines Vierfüsslerrumpfes aus weissem Kalkstein.

2) Annual of the British School at Athens No. IX, Pl. X.

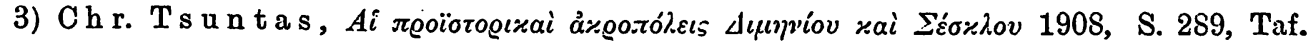
33, 2, 7; Taf. 37, 1 . 

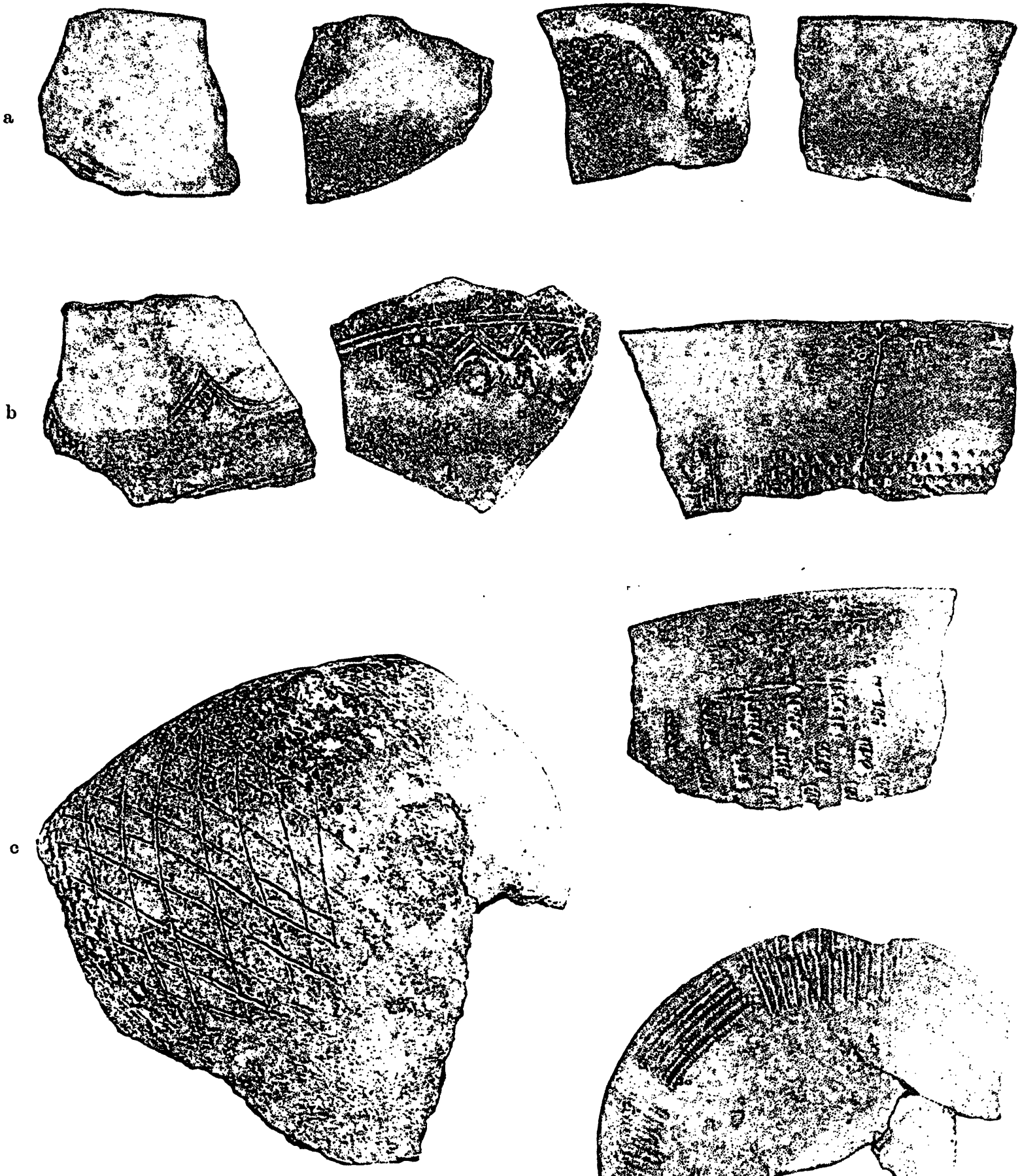

Scherben von Tongefässen a) mit roter Mattmalecei, b) mit eingetieften Ornamenten, c) mit Dekoration im sog. Rahmenstil, d) in (ler ,.Stichkanaltcchnik“, 1/2 nat. (ir. - National-Nluseuın in Belgrarl.

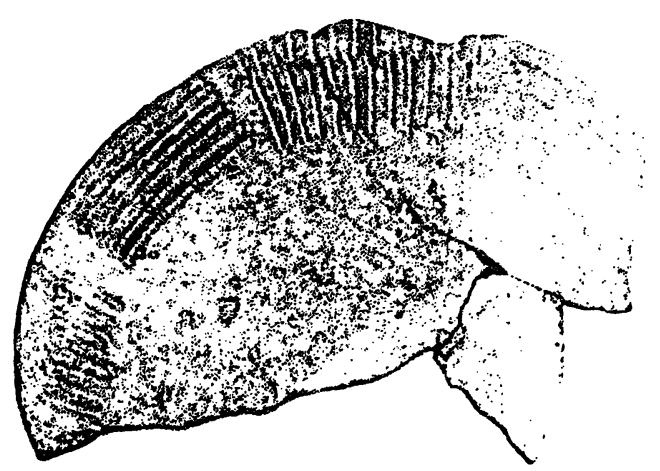



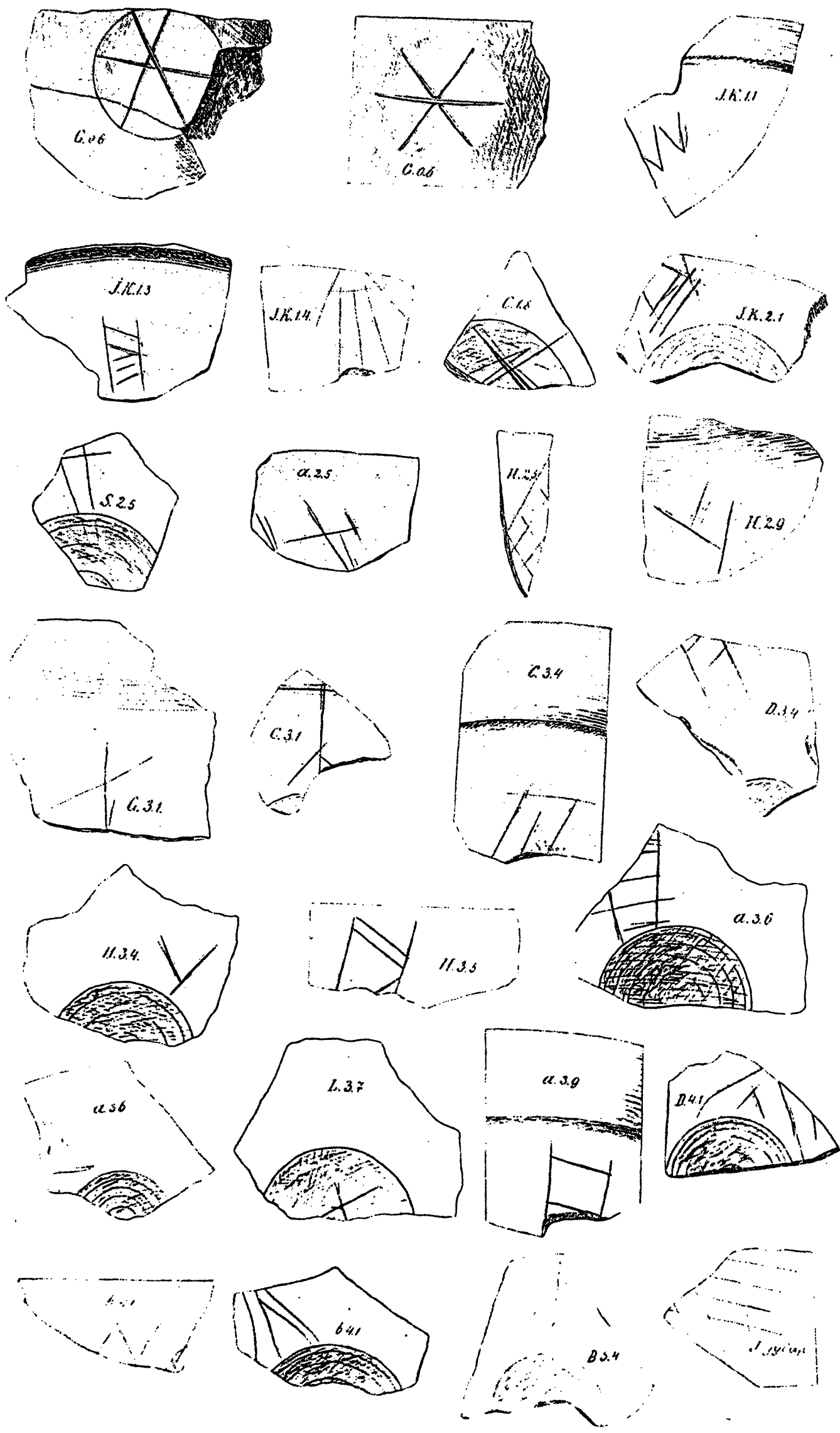

Tongefässscherben mit pietagraphischen Zcichen oder Marken, $1 / 2$ nat. Gir - National-Muscum in Belgrad 
Für weitere Klassifikationen der plastischen Darstellungen des menschlichen Körpers in Serbien ebenso wie in Vinča selbst verweisen wir auf unseren Artikel „South-Eastern Elements in the Pre-historic Civilization of Servia" in Annual of the British School at Athens Nr. XIV, S. 322 ff., und an dieser Stelle wollen wir in Wort und Bild nur noch einige Ergänzungen zu unseren früheren Ausführungen liefern.

Die Darstellungen Taf. 9, a-g zeigen uns die drei Entwicklungsstufen der Vinčaer Plastik in ihrer stratigraphischen und wohl auch zeitlichen Folge. Die Hauptmerkmale dieser drei Stufen sind folgende:

An dem Exemplare Taf. 9, a sehen wir ein mehr oder weniger dreieckiges Gesicht mit einer warzenförmigen Erhöhung, etwa in der Mitte des Dreieckes, welche die Nase darstellt. Ausgenommen die Darstellung der Brüste, sind keine weiteren Angaben an den Statuetten dieses Typus vorhanden. Derartige Darstellungen rechnen wir zum ältesten Typus, welcher in den untersten Strata in Vinča erscheint und welchen wir als ,ägäisch" oder ,,kykladisch" im Sinne der Definiton des“Herrn J o h n L. M y re s ${ }^{1}$ ) bezeichnen möchten: Dieser ägäische Typus, nach der Klassifikation von Herrn L a g r a n g e, entspricht den Erscheinungen in Early Minoan III (E. M. III) ${ }^{2}$ ) in Knossos, ohne mit ihnen etwa gleichzeitig zu sein.

Den entwickelten Typus (Taf. 9, b-f), der in Vinča hauptsächlich den mittleren Strata angehört, charakterisieren detailiertere Darstellungen am Kopfe ebenso wie am übrigen Körper der Statuetten: Die Darstellung der Haare (in beiden Variationen: durch die vertikalen Löcher am Absatze und durch die eingeritzten Linien), der Augen (plastisch und gezeichnet), der tätowierten und gemalten Muster am Gesichte, der Tracht am Körper, des Körperschmuckes (Halsband und Schulterschmuck) und des Schuhwerkes. Dieser Typus dürfte dem entwickelteren kykladischen Statuettentypus $^{3}$ ) und etwa auch den Figuren von Petsofa (MI. M. I) ${ }^{4}$ ) entsprechen.

Der dritte oder degenerierte Typus wird gekennzeichnet durch die verkümmerten Darstellungen einzelner Erscheinungen der beiden vorhergehenden Typen und das bekannte „Vogelgesicht" mit der stark betonten und vorspringenden Nase (Taf. 9, g). Bezeichnend ist für diesen Typus auch sein Vorkommen in den oberen und obersten Strata von Vinča, wie auch an den diesen Strata entsprechenden Fundorten in Serbien.

Der neue, dem Kličevacer Idol in vieler Hinsicht entsprechende Statuettentypus in Vinča ist vertreten durch einen zufälligen Fund von Vinča, welcher zugleich auch für die serbischen Fundorte der jüngeren Gruppe bezeichnend ist. Er ist, wie an anderer Stelle gezeigt werden soll, von den Erscheinungen der mykenischen Periode abhängig. Abb. S. 101.

1) B. S. A. Nr. IX, S. 366 .

2) La Crète ancienne, 1908, S. 77 ff.

3) Athenische Jtitteilungen XVI, S. 46; J. D \& chel tet te, Manuel d'archéologie préhistorique, 1908, S. 597, Abb. 230, 1.

1) B. S. A. IX, S. 360 ff., Pl. VIII-XIII; D. M a ckenzie, B. S. A. Nr. XII, S. 233, 245; R. II. Burrow s, Discoveries in Crete, 1908, S. 53. 
Zu den $K$ nochenstat uot te $n$ in Taf. 9, $i$ sind zunächst zu erwälnen Analogien aus Bulgarien, ${ }^{1}$ ) dann aus Troja ${ }^{2}$ ) und schliesslich des Materiales wegen auch eino aus Thessalien, ${ }^{3}$ ) welche ihre nächste Analogie wieder in dem erwähnten Sultan Selo in Bulgarien hat.

Weit grössero Beachtung verdient die thessalische Analogie zu unserer Statuette, welohe einen neuen Typus für Serbien repräsentiert. Dargestellt ist eine auf dem Throne sitzende und das Kind stillende Frau (Kurotrophos). Der Vergleich dieser in Vinča in der Tiefe von 4,50 $\mathrm{m}$ unter der Erdoberfläche gefundenen Figur mit derjenigen aus Thessalien, ${ }^{4}$ ) welche Tsuntas in die "zweite neolithische Periode" datiert, ist sehr lehrreich. Diese thessalische Statuette gehört samt anderen dortigen Beispielen zu den thronenden Figuren, und Tsuntas hat wohl Recht gehabt, sie mit unseren Statuetten dieses Typus zu vergleichen..$^{\text {) }}$ Abb. S. 101.

Zu den A n hä ng se l n aus Marmor und weissem Kalkstein (Taf. 10, a) lassen sich ebenso Analogien aus Südosten anführen, wie z. B. aus Troja ${ }^{6}$ ), Hanai-Tepeh (untere Schicht) ${ }^{7}$ ) und Thessalien. ${ }^{8}$ )

$\mathrm{Zu}$ unseren Muschelringen und Anhängseln aus Muschelschalen (Taf. 10, a.b) sind noch zahlreichere Analogien aus dem Südosten vorhanden. So führen wir einen ganzen Depotfund von 20 Stück solcher Muschelringe im Kreismuseum zu Varna (aus Kozludže in Bulgarien) an, welcher als Depotfund von Bedeutung ist. Anhängsel aus Muschelschalen sind auch in Troja ${ }^{9}$ ) vorhanden und Muschelringe auch aus Thessalien. ${ }^{10}$ ) Es ist aber bekannt, dass Muschelringe auch in Ägypten vorkommen.

Nicht weniger zahlreiche Analogien finden wir im Südosten zu den Erscheinungen an der Vinčaer $\mathrm{K}$ e $\mathrm{r}$ a $\mathrm{m}$ i k im engeren Sinne des Wortes. Doch sollen hier nur einige Hauptpunkte hervorgehoben werden.

Von den Gefässformen kommen die Analogien zu unserer F u s s s c h a le (Taf. 11, a) in Betracht, welche in derselben Form zunächst in Tordos, dann weiter in Bulgarien ${ }^{11}$ ) und schliesslich in Thessalien ${ }^{12}$ ) vorkommt. Als zufälliger

1) Vgl. Bulletin de Correspondance héllénique, t. XXX (1906), S. 415, Abb. 57 ; eine weit grössere Zahl derselben in verschiedenen Entwicklungsstufen und Typen sind uns aus $\mathrm{S}$ u $\mathbf{l}$ t a $n$ Selo in Bulgarien bekannt, welche Herr Anastas Čilingirov demnächst reröffentlichen wird. (Inzwischen ist diese Publikation schon erschienen in ,Sbornik za narodni umotvorenia, nauka i knižnina“, Bd. XXV (1910) unter dem Titel „Knochenidole von der prähistorischen Ansiedlung beim Dorfe Sultan“ S. 1-54 und 3 Tafeln.)

2) Katalog Nr. 7623 und 7624 .

3) Tsuntas, op. cit. S. 306 , Abb. $230 \delta$.

4) Tsuntas, op. cit. S. 290 , Taf. $31, \mathrm{Nr} .2$.

5) Op. cit. S. 379 .

6) Katalog Nr. $7763 \mathrm{ff}$.

7) R. Virchow, Alttrojanische Gräber und Schädel, Abhandlungen der Königlichen Akademie der Wissenschaften zu Berlin aus dem Jahre 1882, S. $77 \mathrm{ff}$.

8) Tsuntas, op. cit. S. $337 \mathrm{ff}$., Taf. 43 , Nr. $7 \mathrm{ff}$.

9) Katalog Nr. 7772-7773; 7802.

10) Tsuntas, op. cit. S. 356 , Taf. 46 , Nr. 5-7.

11) Aus Topra-Asar, Periodičesko Spisanie, 1908, Sophia, S. 380, Abb. 20.

12) Tsuntas, op. cit. S. 213, Taf. 23, Nr. 3, 4; S. 222, Taf. 10, Nr. 1, 2. 
Fund ist einmal auch ein hoher hohler Fuss in Vinča ${ }^{1}$ ) gefunden worden, so dass man berechtigt ist, zu schliessen, dass in Vinča auch „oben sich schliessende, bauchige Gefässe.... teils kugelbauchig, teils mit Umbruch der Wandung ..."2) bekannt waren, dessen südöstliche Exemplare schon H. S c h m id t aufgezählt hatte. ${ }^{3}$ ) Jedenfalls ist eine Trennung der Schale von den oben sich schliessenden Gefässen mit hohem, voll oder hohl gearbeitetem Fusse nach dem heutigen Stande unserer Kenntnis in Vinča nicht möglich. Wir begnügen uns aber, hier auf diese südöstlichen Analogien hinzurreisen.

Weit grössere Beachtung verdient die anthropomorphe Vase in ihren beiden Varietäten: a) mit der Gesichtsdarstellung auf dem Halse (Abb. 7) und b) auf dem Gefässdeckel (Abb. 8). Genügend bekannt sind die hierher gehörenden Analogien aus Troja II-V ebenso wie die Gesichtsdarstellungen auf den Gefässdeckeln von Tordos. ${ }^{4}$ ) Aber auch in Thessalien ist die anthropomorphe Vase vertreten, obzwar sie, soweit es uns bekannt ist, von keinem Forscher als solche erkannt wurde. Ihr Vorhandensein beweisen uns folgende Funde: 1. das Gefäss auf Taf. 16, Nr. 2, und Gefässfragmente auf Taf. 16, Nr. 1, welche Tsuntas (op. cit. S. 201) seiner ,,zweiten neolithischen Periode" zuschreibt; 2. die vier Deckel (ibid. S. 274, Abb. 199), welche Tsuntas seiner „Bronzeperiode" zuschreibt und mit den ähnlichen Deckeln aus Troja I vergleicht (S. 275, 364), und schliesslich 3. das Fragment in Abb. 228, S. 301, mit der Gesichtsdarstellung, welches Tsuntas gleichfalls seiner „Bronzezeit” zuschreibt (S. 300).

Die zylindrische, sich nach oben verjüngende Halsform an dem Fragmente Taf. 16, Nr. 1, dann die rauh gelassene und unverzierte Halsoberfläche an beiden Beispielen setzt die Gefässdeckel mindestens in der Form voraus, wie dasjenige in Abb. 199, S. 274 ist. Dass aber derartige Gefässe auch die Deckel mit der Gesichtsdarstellung haben konnten, beweist uns das angeführte Fragment in Abb. 228, S. 301, für welches auch Tsuntas selbst sagt, dass es zu den Gefässen und nicht zu den Tonstatuetten gehört (S. 300). Wir sehen in diesem Fragmente den Teil eines Gefässdeckels mit der Gesichtsdarstellung, obzwar Tsuntas bei der Besprechung der Beispiele auf Taf. 16, Nr. 1 und 2, sagt, dass ron derartigen, zu den Gefässen gehörenden Deckeln „,keine Scherbe gefunden wurde" (S. 204). Nach all dem glauben wir behaupten zu können, dass die anthropomorphe Vase auch in Thessalien bekannt war, welche Tatsache sich für die Datierung der Funde aus Thessalien und Vinča verwerten liesse.

Was die Ornamenttechniken betrifft, so finden wir die der eingedrückten Ornamente, die nordwestlich von unseren Fundorten nicht vorzukommen scheint, in südöstlicher Richtung in zwei verschiedenen Stufen wieder: eine jüngere Stufe in Bulgarien (Sultan Selo), dann einerseits in

1) Starinar, N. S. Bd. I, S. 119 , Abb. 36; Mem non Bd. I, S. 185, Abb. 35.

2) H. Sc h m id t, Zeitschrift für Ethnologie, 1904, S. 655.

3) Ibid. S. 65.5 ff. - Vgl. auch J. D é c h e l e $t$ t e, Revue archéolugique 1908, II, S. 250 ff.

4) H. Sc h m id t, Zeitschrift für Ethnologie, 1903, S. 455, Abl. 35. 
Thessalien aus der dortigen Bronzezeit (nach Tsuntas) ${ }^{1}$ ) und andererseits in Troja $\left.(\text { ? })^{2}\right)$, Bosöjük und in der Nekropole Yortan bei Smyrna. ${ }^{3}$ ) Für die Vinc:afunde dieser Technik (Taf. 11, b) ist es bezeichnend, dass sie alle, gleich den aufgezählten südöstlichen Analogien, immer bestimmte Muster, in Tinía selbst die Spirale, aufweisen. Dagegen erscheint die ältere Stufe dieser Technik in Vinca durchaus nicht. Wir kennen diese ältere Stufe nur aus Thessalien, und zwar aus der älteren neolithischen Periode nach Tsuntas, $\left.{ }^{4}\right)$ und von Kreta her. Derartig ausgeführte Vertiefungen auf den kretischen Funden von Knossos ${ }^{5}$ ) und Phaistos $^{6}$ ) gehören der dortigen neolithischen Zeit an und erscheinen als eine natürliche Folge des Glättens der Gefässoberfläche. Sie haben keinen richtigen ornamentalen Charakter. So mangelhaft wie sie ist, die thessalische Stratigraphie erlaubt uns, die Entwicklung dieser Technik in Thessalien in grossen Zügen zu verfolgen. Wir begegnen in ihr einer Erscheinung, welche für die richtigere Beurteilung und das bessere Verständnis einzelner Kulturerscheinungen im Norden der Balkanhalbinsel ebenso wie im Donautale von weittragender Bedeutung sein kann. Uns scheint nämlich, dass sich damit eine uralte Übuing von Kreta aus bis nach Thessalien verpflanzt, wo sie sich unter verschiedenen Einflüssen innerhalb der Zeit und des Raumes verändert und sich als solche auch weiter auf den bewährten Kulturwegen ausbreitet. Nicht viel anders steht es auch mit der anderen ornamentalen $\mathrm{Technik} d$ er geglät teten schwarzen Ornamente auf der grauweiss überzogenen Gefässoberfläche (Taf. $12-13$ ). In Bulgarien ${ }^{7}$ ) ist diese Technik gleichfalls vertreten, und wir begegnen ihr auch in Thessalien, ${ }^{8}$ ) andererseits wieder in Besika-Tepeh ${ }^{9}$ ) und Bos-Öjük. ${ }^{10}$ ) Demnach ist es zu schliessen, dass die Technik der eingedrückten ebenso wie diejenige der geglätteten schwarzen Ornamente fast an gleichen Fundorten, oder besser gesagt in gleichen Gegenden, zusammen und wohl auch gleichzeitig vorkommen. Dies ist namentlich deshalb wichtig, weil wir diese beiden Techniken in Vinča in allen Schichten finden. Diese Tatsache werden wir weiter bei der Datierung der Vinčastrata ausführlich besprechen und $\mathrm{zu}$ verwerten suchen.

Die Technik der rote $\mathrm{n}$ attmalerei ist auf den Beispielen in Taf. 15, a zum ersten Male in Serb̈ien bei der Ausgrabung in Vinča festgestellt. worden. In der Art, wie sie hier vorkommt, stimmt diese Technik, wie es uns scheint, am meisten mit den Techniken, die Tsuntas in Thessalien beobachtet hat und die er der dortigen Bronzezeit zuschreibt, ${ }^{11}$ ) überein. Die Technik

\footnotetext{
1) Tsuntas, op. cit: S. 240 ff. und 372.

2) Katalog Nr. 2263.

3) Tsuntas, op. cit. S. 371, Anm. 1 .

4) Tsuntas, op. cit. S. 161; vgl. auch S. 241 .

5) D. Ma ckenzi e, Journal of hellenic Studies, XXIII, S. 160.

6) Tsuntas, op. cit. S. 242, Anm. 1.

7) Starinar, N. S. Bd. I, Dod atak, S. 8, Abb. 3; vgl. auch S. 19 ff.

8) Tsuntas, op. cit. S. 240, Abb. 139-141; vgl. auch S. $370 \mathrm{ff}$.

9) W. Dörpfeld, Troja und Ilion, S. 546.

10) Tsuntas, op. cit. S. 371, Anm. 1 .

11) Op. cit. S. $244(\Gamma 1 \gamma)$ und S. $247(\Gamma 1 \delta)$.
} 
auf den Stücken aus Vinča unterscheidet sich von denjenigen $(\Gamma 1 \gamma)$ aus Thessalien nur dadurch, dass in Vinča nur rote., in Thessalien aber rote und weisse Farbe für die Ornamente verwendet ist. An den thessalischen Gefässen der Gattung $\Gamma 1 \gamma$. werden die Ornamente nur in weisser Farbe ausgeführt.

Die betreffenden Ornamente in Vinča sind in pastoser roter Farbe an beiden Gefässoberflächen gemalt; die Ornamentsstreifen sind nicht geglättet, und die Farbe selbst lässt sich sehr leicht abreiben und auslöschen. Für die auswärtige Provenienz dieser ornamentalen Technik, die in Vinča dennoch heimisch gew orden und daselbst ausgeübt war, spricht namentlich der Umstand, dass derartige mattrote Ornamente fast ausschliesslich an den Gefässen vorkommen, welche zunächst in den altbewäbrten Techniken - derjenigen der eingedrückten und derjenigen der schwarzen, geglätteten Ornamente - verziert und nachher noch mit den mattroten Ornamenten versehen sind. Es kommt daher nicht selten vor, dass alle diese Techniken zusammen auf einem und demselben Gefässe vereinigt sind; gewöhnlich aber eine von den beiden ersteren mit der Technik der roten Mattmalerei.

Bezeichnend für diese Technik sind zwei andere Umstände: 1. sie tritt in Vinča spät auf, so dass sie zu den übrigen ornamentalen Techniken dort zeitlich in ähnlichem Verhältnisse steht, wie die Technik der thessalischen Gattungen $\Gamma \dot{1} \gamma$ und $\Gamma 1 \delta$ zu den übrigen ornamentalen Techniken dort, und 2. sie erscheint gerade in denjenigen Schichten in Vinča, wo der rote geglättete oder der farbige, mit dem Pinsel aufgetragene rote Überzug aufhört.

Wir sind geneigt, auch diese Technik als vom Südosten herübergenommene zu betrachten, und zwar namentlich wegen der Analogien aus Thessalien, wo wir bis jetzt auch andere Analogien zu unseren Funden festgestellt haben. Das Fehlen der vermittelnden Fundorte steht. solch einer Annahme nicht im Wege, besonders, wenn man die mangelnde Erforschung der dazwischen liegenden Gegenden bedenkt.

Was die Technik der eingeritzten Ornamente in Vinča anlangt, so kommt sie in dreierlei verschiedenen Arten vor. In den tiefsten und tieferen Schichten finden sich die mit dem scharfen Instrumente in den weichen Ton eingeritzten Ornamente (Taf. 14). Derartig ausgeführte Ornamente sind selten und nur in der späteren Entwicklungsstufe mit weisslicher Masse ausgefüllt (Taf. 14, d); in der Regel werden sie leer gelassen. Die zweite, und zwar sicher jüngere Art dieser Technik besteht darin, dass die Ornamente mit dem stumpfen Instrumente in den, wie es scheint, schon etwas getrockneten Ton eingeritzt werden. Diese technische Art erscheint in den oberen Schichten bis zum Ende des Kulturlebens in Vinča. Gleichzeitig mit der zweiten erscheint auch die dritte Art dieser Technik, die unter der Bezeichnung „Furchenstich-" oder ,Stichkanaltechnik" bekannt ist. Diese Art der Technik ist durchaus bedingt durch die Ausfüllung der Ornamente mit der weisslichen Masse, wodurch sie auch erklärt werden kann. Im Donautale findet sich diese Technik fast ausschliesslich an den Gefässen der ersten Eisenzeit, welche Tatsache auch die Stratigraphie von Vinča bestätigt hat. 
Im grossen und ganzen lüsst sich dieselbe Zeitfolge der beiden ersten Arton dieser Technik, das Einritzen in den weichen oder den schon etwas gehärteten Ton, auch in Thessalien beobachten, während, wenn die Zuteilung einzelner in Frage kommender Stücke richtig ist, die dritte Art vor der zweiten in 'Thessalien erscheint. In der ersten Art sind dort die Gefässe der älteren') und der jüngeren Steinzeit ${ }^{2}$ ) verziert, in der zweiten Art die Gefässe der Bronzezeit. $\left.{ }^{3}\right)$ Allein die dritte Art, die „Furchenstich-" oder "Stichkanaltechnik", kommt in Thessalien schon während der zweiten Periode der neolithischen $Z_{\text {Zeit }}^{*}$ ) - also früher wie die zweite Art - vor.

$\mathrm{Zu}$ den schon von $\mathrm{H}$. Schmidt angeführten südöstlichen Analogien $\mathbf{z u}$ den pictographis chen $\mathrm{Z}$ e i c h e n aus Tordos ${ }^{5}$ ) tritt jetzt auch ein Beispiel aus Thessalien hinzu..$^{6}$ ) Ähnliche Zeichen findet man an den Böden, oberhalb derselben, am Bauche und am Halse der Gefässe von Vinča (Taf. 16). Sie erscheinen in Vinča in allen Schichten.

Schliesslich machen wir noch auf eine Analogie aufmerksam, die eine grössere Beachtung verdient. Es ist die Spirale und die Artihrer A n we n d u g zur Verzierung der Gefässe.

Es soll zunächst festgestellt werden, dass die'Spirale, echte wie auch „eckige", als Ornament etwa an der Grenze zwischen dem untersten und dem Anfang des mittleren Stratums auftritt. Diese Tatsache entspricht dem Auftreten der Spirale zunächst in Troja, wo sie in der dritten Periode der II. bis V. Ansiedlung zum ersten Male vorkommen, ${ }^{7}$ ) und dann in Thessalien, wo sie zum ersten Male in der „zweiten neolithischen Periode" erscheint. ${ }^{8}$ )

Auch die Anwendungsart der Spirale zur Verzierung der Gefässe ist dieselbe in Thessalien, Bulgarien und Serbien. Im Grunde genommen sind es entweder einzelne Spiralen oder die zwei ineinander greifenden Spiralen, die sich regelmässig abwickeln. Falls die zu verzierende Fläche in einer oder der anderen Richtung nicht ausreicht, wird die Spirale an den betreffenden Stellen nur gedacht, und es werden nur am betreffenden Raume die Spiralenteile gezeichnet. Derartige Verwendung der Spirale ist gleichfalls in Bulgarien ${ }^{9}$ ) und Thessalien ${ }^{10}$ ) bekannt. Für ältereVinčastrata und die ihnen entsprechenden Fundorte in Serbien ist eine derartige Verwendung der Spirale bezeichnend und herrscht fast ausschliesslich vor. Ebenso scheint es auch in Bulgarien und Thessalien zu sein. Dagegen kommt die Volute oder die fortlaufende

1) T s u n t a s, op. cit. Taf. 14, Nr. 1.

2) Ibid. Taf. $16-19$.

3) Ibicl. S. 250, Abb. 151, S. 252, Abb. 153, S. 254, Abb. 159 usw.

4) Iibd. Taf. 16, Nr. $3 \alpha \beta$; Taf. 18, Nr. 7, Taf. 19, Nr. 3.

5) Zeitschrift für Ethnologie 1903, S. 459.

6) Tsunt a s, op. cit. S. 251 , Abb. 152.

7) W. D ö r p f eld, Troja und Ilion, S. 279.

8) Tsuntas, op. cit. S. 203.

9) Bull. de Corr. hell. t. XXX, S. 411, Abb. 47; S. 413, Abb. 53; Revue archéol. 1901 (XXXIX) S. 342, Abb. 11 a, b; S. 343, Abb. 12 u. 13; S. 347, Abb. 17 b.

10) Tsuntas, op. cit. Taf. 22 u. Taf. 24 , Nr. 2 u. 5 ; Taf. 26 , Nr. 3 ; Taf. 30, Nr. 1. 
Spirale (Butmirstufe) an serbischen Fundorten erst in der Eisenzeit vor. Sie erscheint in Serbien unserer Ansicht nach erst in der Zeit, in welcher auch andere, der mykenischen Epoche (L. M. III) zugehörige Erscheinungen in Serbien auftreten. Falls sich diese Tatsache auch weiter bewahrheitet, gewinnen wir dadurch ein Mittel für die relative Datierung einzelner Funde, bei welchen die Spirale bzw. Volute vorkommt.

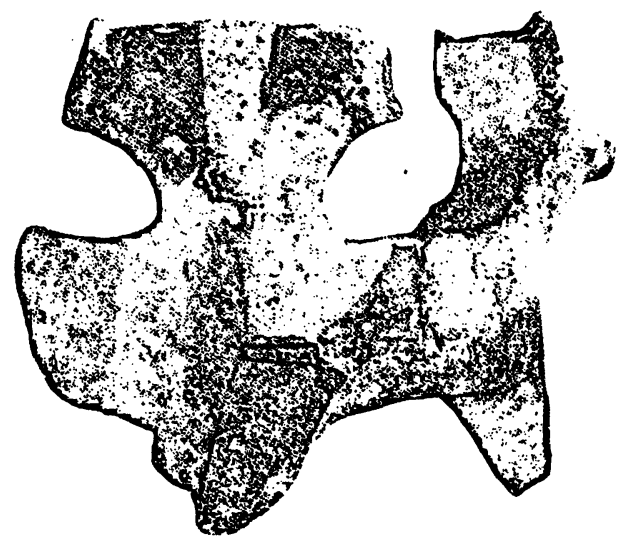

Abb. 9. Dreifüssiges „Altärchen“ von Vinča, etwa $1 / 2$ nat. Gr. - National-illuseum in Belgrad.

(Fortsetzung folgt.)

\section{Eine Renntierhornwaffe aus̀ dem Westhavellande}

\section{Von A. W. Bregger')}

Im Februar dieses Jahres bei einem flüchtigen Durchsehen der grossen Funde von Knochensachen aus dem Havelland, die in der vorgeschichtlichen Abteilung des Königlichen Museums in Berlin aufbewahrt werden, und die ich schon im Frühjahre 1908 Gelegenheit hatte vollständig zu studieren, wurde es mir klar, dass man zur richtigen Würdigung der Bedeutung dieser Stücke, die Verhältnisse kennen musste, unter denen sie gefunden sind. ${ }^{2}$ )

1) Nach dem Mss. übersetzt von MI. Ebert.

2) Die Sachen bestehen im wesentlichen aus folgenden Haupttypen: A. Harpunen mit zwei Reihen Widerhaken, die im allgemeinen abwechselnd gestellt sind. Der Unterteil ist triangulär, abgeflacht. Die Widerhaken rhombisch (Abbildung 1). B. Harpunen mit einer Reihe Widerhaken, im allgemeinen wenige, grosse und rhombische (Abbildung 2-3). C. Harpunen (?) mit einigen wenigen Widerhaken ganz oben an der Spitze, nicht vonoinander geschieden durch einen grösseren Zwischenraum (Abb. 4-5). D. Fine Zwischenform zwischen C und B, vom Charakter wie S. Müller, Ordning, Stenalderen, Abb. 185, viele, dichtstohende Widerhaken. Ausserdem werden einzelne zylindrische Beinspitzen wie Müller, Ordning, Abb. 184 angetroffen. Teiter schwere Angelhaken (?) ohne Widerhaken, von primitirem Charakter, aber von einer aussergewöhnlichen Länge (bis $20 \mathrm{~cm}$ ). Pfriemen von grösseren Knochen, Hornhacken und Hornäxte mit Schaftloch usw. Die meisten von den Berliner Museumsstūcken sind aus dem Havelland, und von verschiedenen Fundplätzen (Döberitz, Pritzerbe, Ferchesar, Fernewerder usw.). Bei der archäologischen Bedeutung dieser Stücke gedenke ich bei einer späteren Gelegenheit darauf zurückzukommen. 